\title{
In-Plane Vibration Analysis of Square Plate with Multiple Cutouts
}

\author{
Shuangxia Shi, ${ }^{1}$ Yinlang Qi, ${ }^{1}$ Changqing Zhang, ${ }^{1}$ Qingtai Meng, ${ }^{1}$ Yinbei Liu, ${ }^{1}$ Guoyong Jin, ${ }^{2}$ \\ and Bin Xiao $\mathbb{D D}^{1}$ \\ ${ }^{1}$ School of Energy and Power Engineering, Northeast Electric Power University, Jilin 132012, China \\ ${ }^{2}$ College of Power and Energy Engineering, Harbin Engineering University, Harbin 150001, China
}

Correspondence should be addressed to Bin Xiao; dr.binxiao@yahoo.com

Received 23 January 2021; Revised 16 March 2021; Accepted 26 April 2021; Published 5 May 2021

Academic Editor: Sumeet S. Aphale

Copyright (C) 2021 Shuangxia Shi et al. This is an open access article distributed under the Creative Commons Attribution License, which permits unrestricted use, distribution, and reproduction in any medium, provided the original work is properly cited.

\begin{abstract}
A theoretical solution method for the in-plane vibration characteristics of the plate with cutouts is proposed in this paper. The energy principle in conjunction with the Rayleigh-Ritz solution technique is adopted for the theoretical modeling of the in-plane vibration of the structure. The energy functions for the plate with cutouts are established by subtracting the energies of the cutout domains from the total energies of the whole plate. To ensure continuity over the entire solution domain, the in-plane displacements are composed of two-dimensional standard Fourier series and supplementary functions. The in-plane eigenmodes of the plates with different square cutouts are compared with the results obtained from the finite element method (FEM), with good agreements. The influences of the cutouts on the in-plane vibration characteristics of the plates with cutouts are investigated by varying the number, size, and position of the cutouts.
\end{abstract}

\section{Introduction}

The plates with cutouts are a kind of structure widely used in engineering fields, such as in the field of aeronautics, marine, and civil engineering. The cutouts within the structures are usually used to facilitate the connection of substructures. The in-plane vibration of the plates could not be ignored in the theoretical modeling for the connected structures; thus, investigating the in-plane vibration characteristics of the plate with cutouts is of great significance in the field of vibration control in engineering practice. If the in-plane vibration characteristics of the plate with cutouts are calculated by the finite element method (FEM), the results are more effective for low frequencies but require high computational cost for high frequencies. To reduce the computation time and reveal the physical mechanism of the influence of the cutouts within the plate on the in-plane vibration, analytical methods are more applicable for predicting the in-plane vibration characteristics of the plate with cutouts.

The number and size of cutouts have a great influence on the transverse vibration characteristics of the plate structures
[1-9]. The rigidity of the structure with cutouts is mostly dependent on the number of cutouts. The vibrations of rectangular plates with one and two cutouts are, respectively, investigated by Avalos [1, 2]. Lam [3] used the Rayleigh-Ritz method to resolve the vibration problems of single opening and double opening structures. Moon [4] is concerned with the vibration analysis of a rectangular plate with a rectangular or circular hole. Lu [5] proposed an analytical solution of an elastic infinite plate containing an ellipse hole and a circle hole subjected to internal tractions and shears at infinity. The investigations on the effects of the cutout size are focused on the cutouts located in the center of the flexible plate [6-9]. Takahashi [6] used the Ritz method to analyze the rectangular plate with a central circular hole. Paramasivam [7] studied the effects of rectangular cutout sizes on fundamental frequencies of the structure with different types of boundary conditions by extending the grid framework model.

By comparison, the theoretical investigations on the inplane vibrations of the plates are less than the transverse vibrations of the flexible plates. The Rayleigh-Ritz method $[10,11]$ and dynamic stiffness method [12-14] are the 
common theoretical methods for solving the vibration problems of structures. The in-plane vibration of isotropic rectangular plates with various boundary conditions is studied by the Rayleigh-Ritz method [10]. Gorman [12, 13] used the superposition method to analyze the in-plane vibration characteristics of the plates with different types of boundary conditions. At present, the researchers pay more attention to the theoretical solutions for the in-plane vibration analysis of the plate with cutouts [15-20]. The natural frequencies and mode shapes of the square plate with an inner cutout are obtained by the discrete Ritz method [16]. Huang [15] used the receptance approach to investigate the in-plane vibration characteristics of plates with circular cutouts. Zhou [18] investigated the free in-plane vibration problem of rectangular plates with classical boundary conditions using various numerical methods. A modified Fourier-Ritz approach is developed to investigate the free in-plane vibration characteristics of the orthotropic circular, annular, and sector plates with general boundary conditions [19]. Shi [20] adopted an improved Fourier series method and the first-order beam theory to study the in-plane free vibration behaviors of functionally graded carbon nanotubereinforced composite circular arches and curved beams under elastic boundary restraints.

In studying the effects of cutouts within the structure, the cutouts are mostly located in the center of the structure, and the number of cutouts is less than two. Comparing with the investigations above, the theory proposed in this paper mainly could be used to predict the in-plane modal characteristics of the plate having arbitrary number and position of cutouts. In the existing methods, the cutouts within the structure are considered as a kind of structure with zero thickness. To behave the physical characteristics of the cutouts within the structure, the influence of cutouts on the vibration characteristic of the structure is considered in terms of energy [21]. The energy principle combined with the Rayleigh-Ritz solution is adopted to determine the vibration characteristics of the structures with cutouts. The effect of the cutouts is taken into account by subtracting the energies of the cutout domains from the total energies of the whole plate. The modal characteristics of square plates with different cutouts are predicted by the present method and verified by FEM. In the Results and Discussion, the influences of the number, size, and position of the cutouts on the natural frequencies and mode shapes of the plate with cutouts are investigated.

While previous researches focused mainly on relatively simple sandwich constructions and approximated the ribstiffeners as an Euler-beam or a combination of translational spring and rotational spring, an exact theoretical model concerning the vibroacoustic response of more complex structures (e.g., two-dimensional sandwich structures orthogonally reinforced by periodic rib-stiffeners) is desirable. In addition to helping to explore the underlying physical subtleties, the model should also serve as benchmark checking for approximate analytical approaches, with a small computational expense afforded compared to numerical methods such as the FEM and the boundary element method (BEM).

\section{Theoretical Formulations}

2.1. Description of the Numerical Model. The geometric sketch of the plate with cutouts investigated in this paper is given in Figure 1. The boundary conditions of the rectangular plate with cutouts shown in Figure 1 can be represented by two kinds of springs with determined stiffness constants. The linear springs are attached to the edges in the normal or tangential direction. The dimensions in $x$ and $y$ directions for the rectangular plate with cutouts are represented as $a$ and $b$, respectively. The rectangular cutouts positions are unrestricted in Figure 1. The length and width of the $i$ th cutout are, respectively, $(X d i-X c i)$ and $(Y d i-Y c i)$, in which the size of the rectangular cutout is variable.

When the in-plane displacements of the rectangular plate are periodically extended as a standard Fourier series on the $x-y$ surface, the discontinuities problems for the original displacement functions and their derivatives will encounter along the plate structure boundary. Therefore, the in-plane displacement functions of the plate structure are expressed as the modified Fourier series [22] to overcome the potential discontinuity of the original displacement functions and their derivatives. The in-plane displacement functions $\mathrm{u} 1$ in the $x$ direction and v1 in the $y$ direction for the rectangular plate are, respectively, expressed as

$$
\begin{aligned}
u(x, y)= & \sum_{m=0}^{\infty} \sum_{n=0}^{\infty} B_{m n} \cos \lambda_{a m} x \cos \lambda_{b n} y \\
& +\sum_{m=0}^{\infty}[\underbrace{a_{i n m} \xi_{b}(y)}_{y=0}+\underbrace{b_{i n m} \xi_{b}(y)}_{y=b}] \cos \lambda_{a m} x \\
& +\sum_{n=0}^{\infty}[\underbrace{c_{i n n} \xi_{1 a}(x)}_{x=0}+\underbrace{d_{i n n} \xi_{2 a}(x)}_{x=a}] \cos \lambda_{b n} y, \\
v(x, y)= & \sum_{m=0}^{\infty} \sum_{n=0}^{\infty} C_{m n}^{C_{m} \cos \lambda_{a m} x \cos \lambda_{b n} y} \\
& +\sum_{m=0}^{\infty}[\underbrace{e_{i n m} \xi_{1 b}(y)}_{y=0}+\underbrace{f_{i n m} \xi_{2 b}(y)}_{y=b}] \cos \lambda_{a m} x \\
& +\sum_{n=0}^{\infty}[\underbrace{g_{i n n} \xi_{1 a}(x)}_{x=0}+\underbrace{h_{i n n} \xi_{2 a}(x)}_{x=a}] \cos \lambda_{b n} y,
\end{aligned}
$$

where $\begin{aligned} & \lambda_{a m}=m \pi / a, \\ & \lambda_{b n}=n \pi / b,\end{aligned}$ and the supplementary functions in the in-plane displacement functions are expressed as follows:

$$
\begin{aligned}
& \xi_{1 a}(x)=a \zeta_{x}\left(\zeta_{x}-1\right)^{2}, \\
& \xi_{2 a}(x)=a \zeta_{x}^{2}\left(\zeta_{x}-1\right), \quad\left(\zeta_{x}=x / a\right), \\
& \xi_{1 b}(y)=b \zeta_{y}\left(\zeta_{y}-1\right)^{2}, \\
& \xi_{2 b}(y)=b \zeta_{y}^{2}\left(\zeta_{y}-1\right), \quad\left(\zeta_{y}=y / b\right) .
\end{aligned}
$$

It can be inferred that the supplementary functions are introduced to remove all the discontinuities potentially 
associated with the original displacement and their derivatives throughout the entire solution domain including the boundaries and then to effectively enhance the convergence of the results.

2.2. Solution Procedure of the Plate with Cutouts. Among the solutions of vibration analysis for the continuous systems, the energy principle combined with the Rayleigh-Ritz method is often adopted to solve the vibration characteristics of the rectangular plate due to the reliability and efficiency of the results $[11,22]$. The energy function of the plate with cutouts is obtained by deleting the potential and kinetic energies on the cutout domains from the energy functions of the whole plate. Therefore, the Lagrange function for the rectangular plate with $n$ cutouts is given as

$$
\begin{aligned}
L= & \left(V-V_{1}-V_{2}-\cdots-V_{i}-\cdots V_{n}\right) \\
& -\left(T-T_{1}-T_{2}-\cdots-T_{i}-\cdots T_{n}\right),
\end{aligned}
$$

where $V$ and $T$ denote the potential and kinetic energy of the rectangular plate without cutouts, respectively. $V_{i}$ and $T_{i}$ denote the potential and kinetic energy of the rectangular plate occupying the $i$ th cutout, respectively.

The potential energy of the rectangular plate without cutouts can be expressed as

$$
\begin{aligned}
V= & \frac{G}{2} \int_{0}^{a} \int_{0}^{b}\left\{\left(\frac{\partial u}{\partial x}+\frac{\partial v}{\partial y}\right)^{2}-2(1-\mu) \frac{\partial u}{\partial x} \frac{\partial v}{\partial y}+\frac{1-\mu}{2}\left(\frac{\partial u}{\partial x}+\frac{\partial v}{\partial y}\right)^{2}\right\} \mathrm{d} x \mathrm{~d} y \\
& +\frac{1}{2} \int_{0}^{b}\left[k_{n x 0} u^{2}+k_{p x 0} v^{2}\right]_{x=0} \mathrm{~d} y+\frac{1}{2} \int_{0}^{b}\left[k_{n x 1} u^{2}+k_{p x 1} v^{2}\right]_{x=a} \mathrm{~d} y+\frac{1}{2} \int_{0}^{a 1}\left[k_{n y 0} v^{2}+k_{p y 0} u^{2}\right]_{y=0} \mathrm{~d} x+\frac{1}{2} \int_{0}^{a 1}\left[k_{n y 1} v^{2}+k_{p y 1} u^{2}\right]_{y=b} \mathrm{~d} y
\end{aligned}
$$

The kinetic energy of the rectangular plate without cutouts can be expressed as

$$
\begin{aligned}
T & =\frac{1}{2} \int_{0}^{a} \int_{0}^{b} \rho h\left[\left(\frac{\partial u}{\partial t}\right)^{2}+\left(\frac{\partial v}{\partial t}\right)^{2}\right] \mathrm{d} x \mathrm{~d} y \\
& =\frac{1}{2} \rho h \omega^{2} \int_{0}^{a} \int_{0}^{b}\left(u^{2}+v^{2}\right) \mathrm{d} x \mathrm{~d} y,
\end{aligned}
$$

where $k_{n y 0}$ and $k_{p y 1}$ represent the spring stiffness acting in the normal direction along the edge of $y=0$ and the spring stiffness acting in the tangential direction along the edge of $y=b$, respectively. $k_{n x 0}$ and $k_{p x 1}$ represent the spring stiffness in the normal direction along the edge of $x=0$ and the spring stiffness acting in the tangential direction along the edge of $x=a$, respectively. $\omega$ is the circular frequency. $\mu$ and $\rho$ denote Poisson's ratio and mass density of the rectangular plate, respectively. $h$ denotes the thickness of the rectangular plate.

The potential energy of the $i$ th cutout within the plate is expressed as

$$
\begin{aligned}
V_{i}= & \frac{G}{2} \int_{X c i}^{X d i} \int_{Y c i}^{Y d i}\left\{\left(\frac{\partial u}{\partial x}+\frac{\partial v}{\partial y}\right)^{2}-2(1-\mu) \frac{\partial u}{\partial x} \frac{\partial v}{\partial y}+\frac{1-\mu}{2}\left(\frac{\partial u}{\partial x}+\frac{\partial v}{\partial y}\right)^{2}\right\} \mathrm{d} x \mathrm{~d} y+\frac{1}{2} \int_{Y c i}^{Y d i}\left[k_{n x 0} u^{2}+k_{p x 0} v^{2}\right]_{x=X c i} \mathrm{~d} y \\
& +\frac{1}{2} \int_{Y c i}^{Y d i}\left[k_{n x 1} u^{2}+k_{p x 1} v^{2}\right]_{X=X d i} \mathrm{~d} y+\frac{1}{2} \int_{X c i}^{X d i}\left[k_{n y 0} v^{2}+k_{p y 0} u^{2}\right]_{y=Y c i} \mathrm{~d} x+\frac{1}{2} \int_{X c i}^{X d i}\left[k_{n y 1} v^{2}+k_{p y 1} u^{2}\right]_{y=Y d i} \mathrm{~d} y
\end{aligned}
$$

The kinetic energy of the $i$ th cutout within the plate is expressed as

$$
\begin{aligned}
T_{i} & =\frac{1}{2} \int_{X c i}^{X d i} \int_{Y c i}^{Y d i} \rho h\left[\left(\frac{\partial u}{\partial t}\right)^{2}+\left(\frac{\partial v}{\partial t}\right)^{2}\right] \mathrm{d} x \mathrm{~d} y \\
& =\frac{1}{2} \rho h \omega^{2} \int_{X c i}^{X d i} \int_{Y c i}^{Y d i}\left(u^{2}+v^{2}\right) \mathrm{d} x \mathrm{~d} y .
\end{aligned}
$$

The boundary condition for the cutout in the rectangular plate is sensible with its geometric positions. If the cutout is located in the rectangular plate, then the boundary condition of the cutout is free. When the cutout is located on the edge of the rectangular plate, the edge of the boundary condition of the cutout will be the same as that of the rectangular plate.

Substituting equations (5)-(8) into equation (4) and applying the Rayleigh-Ritz procedure against each of the 
unknown Fourier series coefficients, the linear system in the where matrix form can be described as

$$
\left(K-\left(\sum_{i=1}^{n} K_{i}\right)\right)-\omega^{2}\left(M-\left(\sum_{i=1}^{n} M_{i}\right)\right) E=0,
$$

$$
\begin{aligned}
& K=\left[\begin{array}{cccccc}
K_{1-1} & K_{1-2} & K_{1-3} & K_{1-4} & \ldots & K_{1-10} \\
K_{2-1} & K_{2-2} & K_{2-3} & K_{2-4} & \ldots & K_{2-10} \\
K_{3-1} & K_{3-2} & K_{3-3} & K_{3-4} & \ldots & K_{3-10} \\
K_{4-1} & K_{4-2} & K_{4-3} & K_{4-4} & \ldots & K_{4-10} \\
\vdots & \vdots & \vdots & \vdots & \ddots & \vdots \\
K_{10-1} & K_{10-2} & K_{10-3} & K_{10-4} & \ldots & K_{10-10}
\end{array}\right], \\
& M=\left[\begin{array}{cccccc}
M_{1-1} & M_{1-2} & M_{1-3} & M_{1-4} & \ldots & M_{1-10} \\
M_{2-1} & M_{2-2} & M_{2-3} & M_{2-4} & \ldots & M_{2-10} \\
M_{3-1} & M_{3-2} & M_{3-3} & M_{3-4} & \ldots & M_{3-10} \\
M_{4-1} & M_{4-2} & M_{4-3} & M_{4-4} & \ldots & M_{4-10} \\
\vdots & \vdots & \vdots & \vdots & \ddots & \vdots \\
M_{10-1} & M_{10-2} & M_{10-3} & M_{10-4} & \ldots & M_{10-10}
\end{array}\right], \\
& K_{i}=\left[\begin{array}{cccccc}
K_{1-1}^{i} & K_{1-2}^{i} & K_{1-3}^{i} & K_{1-4}^{i} & \ldots & K_{1-10}^{i} \\
K_{2-1}^{i} & K_{2-2}^{i} & K_{2-3}^{i} & K_{2-4}^{i} & \ldots & K_{2-10}^{i} \\
K_{3-1}^{i} & K_{3-2}^{i} & K_{3-3}^{i} & K_{3-4}^{i} & \ldots & K_{3-10}^{i} \\
K_{4-1}^{i} & K_{4-2}^{i} & K_{4-3}^{i} & K_{4-4}^{i} & \ldots & K_{4-10}^{i} \\
\vdots & \vdots & \vdots & \vdots & \ddots & \vdots \\
K_{10-1}^{i} & K_{10-2}^{i} & K_{10-3}^{i} & K_{10-4}^{i} & \ldots & K_{10-10}^{i}
\end{array}\right] \text {, } \\
& M_{i}=\left[\begin{array}{cccccc}
M_{1-1}^{i} & M_{1-2}^{i} & M_{1-3}^{i} & M_{1-4}^{i} & \ldots & M_{1-10}^{i} \\
M_{2-1}^{i} & M_{2-2}^{i} & M_{2-3}^{i} & M_{2-4}^{i} & \ldots & M_{2-10}^{i} \\
M_{3-1}^{i} & M_{3-2}^{i} & M_{3-3}^{i} & M_{3-4}^{i} & \ldots & M_{3-10}^{i} \\
M_{4-1}^{i} & M_{4-2}^{i} & M_{4-3}^{i} & M_{4-4}^{i} & \ldots & M_{4-10}^{i} \\
\vdots & \vdots & \vdots & \vdots & \ddots & \vdots \\
M_{10-1}^{i} & M_{10-2}^{i} & M_{10-3}^{i} & M_{10-4}^{i} & \ldots & M_{10-10}^{i}
\end{array}\right] \text {, } \\
& E=\left\{U^{T} V^{T}\right\}^{T}, \\
& U=\left\{B_{00}, B_{01}, \ldots B_{m 0}, B_{m 1}, \ldots B_{m n}, \ldots B_{M N}, a_{10}, \ldots a_{1 M}, b_{10}, \ldots b_{1 M}, c_{10}, \ldots c_{1 N}, d_{10}, \ldots d_{1 N}\right\}^{T}, \\
& V=\left\{C_{00}, C_{01}, \ldots C_{m 0}, C_{m 1}, \ldots C_{m n}, \ldots C_{M N}, e_{10}, \ldots e_{1 M}, f_{10}, \ldots f_{1 M}, g_{10}, \ldots g_{1 N}, h_{10}, \ldots h_{1 N}\right\}^{T} \text {, }
\end{aligned}
$$

where $\mathrm{K}$ is the stiffness matrix of the structure studied, $M$ is the mass matrix of the structure studied, $K_{\mathrm{i}}$ is the stiffness matrix of the $i$ th cutout within the plate, and $M_{\mathrm{i}}$ is the mass matrix of the $i$ th cutout within the plate. In the numerical calculations, the Fourier series is truncated to
$m=M$ and $n=N$. Once the coefficient vector $E$ is obtained from the eigenvalue equation, the in-plane displacement of the rectangular plate with cutouts can be easily derived by substituting the coefficient vector into equations (1) and (2). 


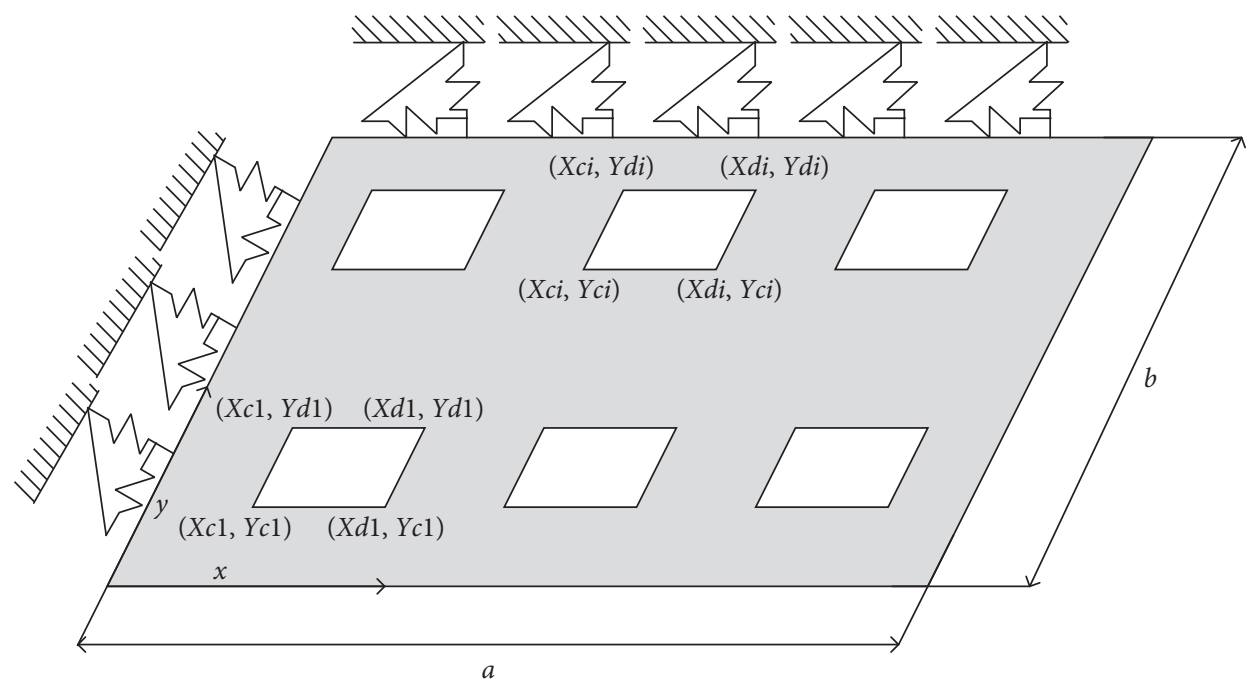

FIgURE 1: The geometric sketch of the rectangular plate with cutouts.

\section{Results and Discussion}

3.1. Convergence and Validation. The convergence of the theoretical modeling is studied by taking a square plate with an inner cutout as an example. The material properties of the square plate with clamped boundary conditions are taken as follows: length $(a)=3 \mathrm{~m}$, thickness $(h)=0.0025 \mathrm{~m}$, Poisson's ratio $(v)=0.33$, density $(\rho)=2700 \mathrm{~kg} / \mathrm{m}^{3}$, and Young's modulus $(E)=7 \times 10^{10} \mathrm{~N} / \mathrm{m}^{2}$. As shown in Figure 1 , the size and original point of the inner cutout are $(0.4 \mathrm{~m} \times 0.4 \mathrm{~m})$ and $(2.2 \mathrm{~m}, 2.2 \mathrm{~m})$, respectively. The modal characteristics for the plate with an inner cutout are given in Table 1 and Figure 2. The simulation results of the rectangular plate with an inner cutout are achieved from the software ANSYS, and the model is constructed by setting the unit size of PLANE42 as $0.05 \mathrm{~m} \times 0.05 \mathrm{~m}$. It can be seen from Table 1 that the natural frequencies for the rectangular plate with an inner cutout are verified by the comparisons with the results obtained from FEM and converge at $M=N=40$. The phenomenon could be seen from the comparisons of mode shapes in Figure 2 that the mode shapes calculated by the current method are wellvalidated.

3.2. The Influence of the Cutout Parameters. In this section, the influence of the number, size, and location of the cutouts on the in-plane vibration characteristics is investigated. In the parametric studies, the material properties and sizes of the square plates with clamped boundary conditions remain unchanged. The length, width, and thickness of the selected plate are $a=40 \mathrm{~m}, b=40 \mathrm{~m}$, and $h=0.0025 \mathrm{~m}$. The material properties of the selected plate are considered as Poisson's ratio $(v)=0.33, \rho=2700 \mathrm{~kg} / \mathrm{m}^{3}$, and Young's modulus $(E)=$ $7 \times 10^{10} \mathrm{~N} / \mathrm{m}^{2}$. Three plate structures with different numbers of cutouts are selected for the investigation on the influence of the number of cutouts. For the rectangular plate with four same cutouts having free boundary conditions, the coordinate positions of the origins for the square cutouts whose size is $5 \mathrm{~m} \times 5 \mathrm{~m}$ are $(12.5 \mathrm{~m}, 5 \mathrm{~m}),(22.5 \mathrm{~m}, 5 \mathrm{~m}),(12.5 \mathrm{~m}$, $30 \mathrm{~m})$, and $(22.5 \mathrm{~m}, 30 \mathrm{~m})$, respectively. For the rectangular plate with six same cutouts having free boundary conditions, the original points of the six cutouts whose size is $5 \mathrm{~m} \times 5 \mathrm{~m}$ are $(5 \mathrm{~m}, 5 \mathrm{~m}),(17.5 \mathrm{~m}, 5 \mathrm{~m}),(30 \mathrm{~m}, 5 \mathrm{~m}),(5 \mathrm{~m}, 30 \mathrm{~m})$, $(17.5 \mathrm{~m}, 30 \mathrm{~m})$, and $(30 \mathrm{~m}, 30 \mathrm{~m})$, respectively. For the rectangular plate with eight same cutouts having free boundary conditions, the coordinate positions of the origins for the cutouts with $5 \mathrm{~m} \times 5 \mathrm{~m}$ size are $(4 \mathrm{~m}, 5 \mathrm{~m}),(13 \mathrm{~m}$, $5 \mathrm{~m}),(22 \mathrm{~m}, 5 \mathrm{~m}),(31 \mathrm{~m}, 5 \mathrm{~m}),(4 \mathrm{~m}, 30 \mathrm{~m}),(13 \mathrm{~m}, 30 \mathrm{~m})$, $(22 \mathrm{~m}, 30 \mathrm{~m})$, and $(31 \mathrm{~m}, 30 \mathrm{~m})$, respectively. The natural frequencies and mode shapes for the rectangular plate with different numbers of cutouts are shown in Table 2 and Figures 3-5, respectively. It can be seen from Table 2 and Figures 3-5 that the natural frequencies and mode shapes for the three cases obtained by the present method are in good agreement with those obtained by FEM. It can be found by the comparison in Table 3 that the more the number of cutouts, the lower the natural frequencies. The reason lies in that the increase of the cutouts makes the stiffness of the plate structure and the natural frequencies decrease. The comparison results between three cases in Figures 3-5 show that the mode shapes are influenced by the number of cutouts within the square plate.

As the in-plane modal characteristics of the structure are influenced by the cutout sizes, three cases of plates with an inner cutout are considered in the numerical discussions. The physical parameters for the plate structure discussed in this section remain unchanged. The sizes of the inner cutouts whose original points are $(5 \mathrm{~m}, 25 \mathrm{~m})$, $(5 \mathrm{~m}, 15 \mathrm{~m})$, and $(5 \mathrm{~m}, 10 \mathrm{~m})$ are $(10 \mathrm{~m} \times 10 \mathrm{~m})$, $(20 \mathrm{~m} \times 20 \mathrm{~m})$, and $(25 \mathrm{~m} \times 25 \mathrm{~m})$, respectively. The natural frequencies and mode shapes for the selected three cases 
TABLE 1: The natural frequencies of the rectangular plate with an inner cutout.

\begin{tabular}{|c|c|c|c|c|c|c|}
\hline Mode & $M=N=30$ & $M=N=32$ & $M=N=34$ & $M=N=36$ & $M=N=40$ & FEM \\
\hline 1 & 978.6 & 978.3 & 978.0 & 977.7 & 977.3 & 973.98 \\
\hline 2 & 1003.4 & 1003.4 & 1003.4 & 1003.4 & 1003.4 & 1003.3 \\
\hline 3 & 1162.6 & 1162.5 & 1162.5 & 1162.4 & 1162.3 & 1161.9 \\
\hline 4 & 1453.6 & 1453.3 & 1453.0 & 1452.8 & 1452.5 & 1449.8 \\
\hline 5 & 1634.8 & 1634.4 & 1634.2 & 1634.0 & 1633.6 & 1631.1 \\
\hline 6 & 1637.7 & 1637.5 & 1637.5 & 1637.4 & 1637.2 & 1636.5 \\
\hline 7 & 1660.3 & 1660.3 & 1660.3 & 1660.3 & 1660.3 & 1660.2 \\
\hline 8 & 1921.5 & 1921.3 & 1921.1 & 1920.9 & 1920.6 & 1918.7 \\
\hline 9 & 2013.1 & 2013.0 & 2013.0 & 2012.9 & 2012.8 & 2012.3 \\
\hline 10 & 2023.6 & 2023.2 & 2023.0 & 2022.6 & 2022.1 & 2018.6 \\
\hline 20 & 2737.2 & 2737.0 & 2736.9 & 2736.8 & 2736.6 & 2735.6 \\
\hline 50 & 3939.1 & 3938.7 & 3938.5 & 3938.2 & 3937.9 & 3937.0 \\
\hline 100 & 5489.0 & 5488.3 & 5488.1 & 5488.0 & 5487.8 & 5488.2 \\
\hline
\end{tabular}
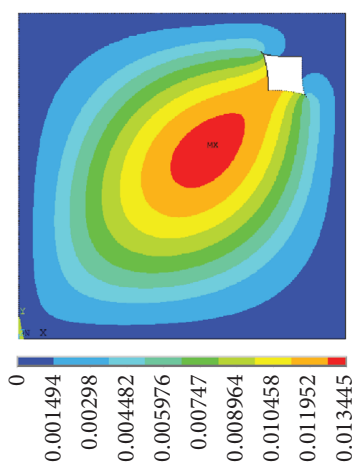

(a)

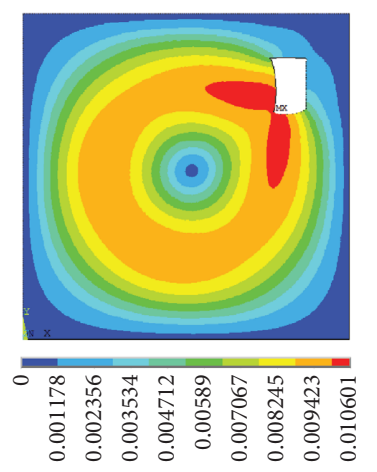

(c)
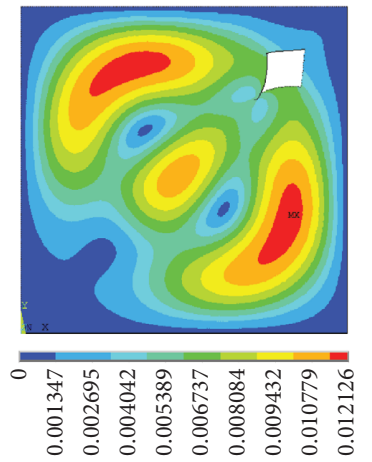
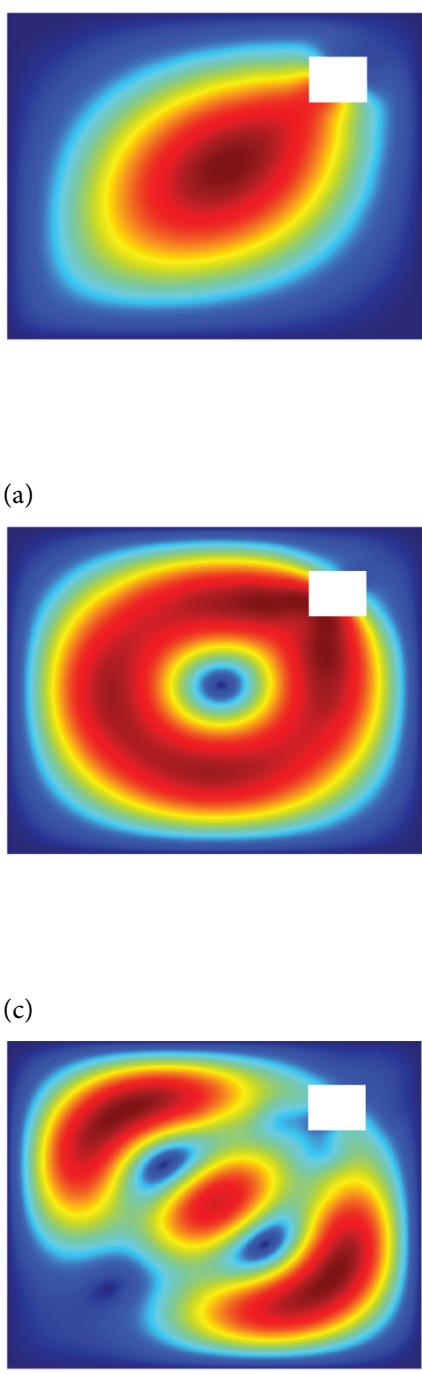

(e)
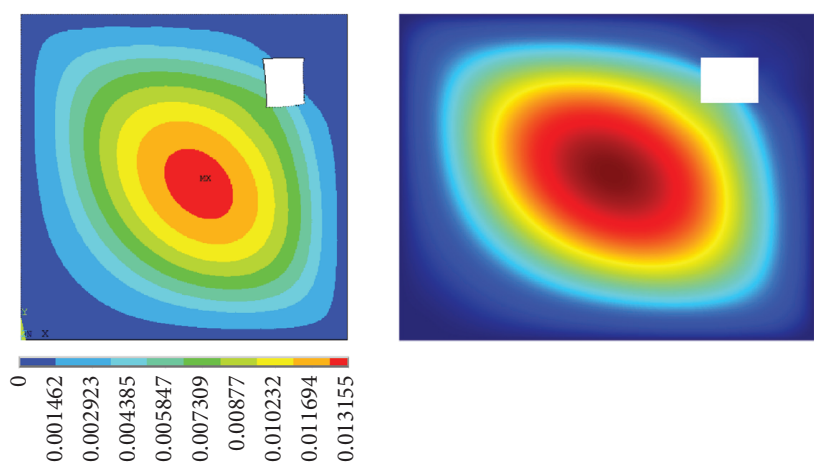

(b)
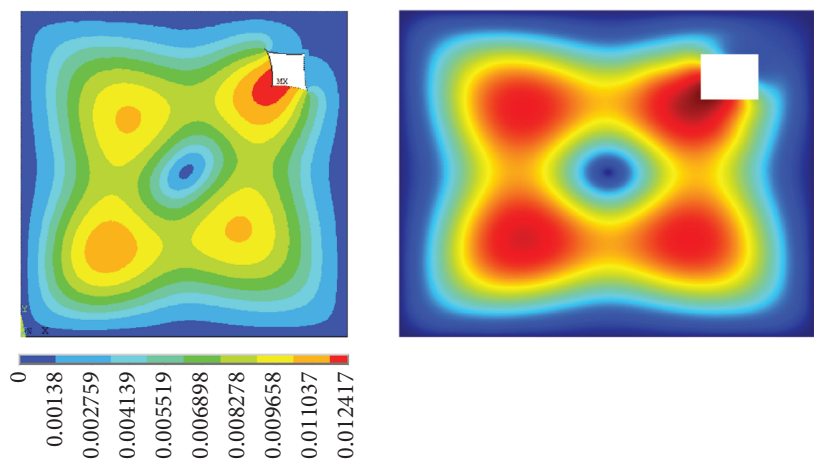

(d)

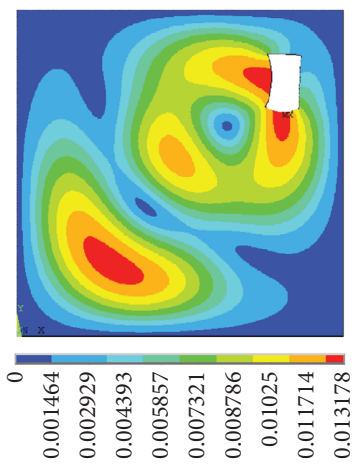

(f)

FIgUre 2: The mode shapes of the square plate with an inner cutout. (a) The first mode shape; (b) the second mode shape; (c) the third mode shape; (d) the fourth mode shape; (e) the fifth mode shape; (f) the sixth mode shape. Left: the ANSYS results; right: the present results. 
TABLE 2: The natural frequencies of rectangular plates with different numbers of cutouts.

\begin{tabular}{|c|c|c|c|c|c|c|}
\hline \multirow{2}{*}{ Mode number } & \multicolumn{2}{|c|}{ Four cutouts } & \multicolumn{2}{|c|}{ Six cutouts } & \multicolumn{2}{|c|}{ Eight cutouts } \\
\hline & Present & FEM & Present & FEM & Present & FEM \\
\hline 1 & 66.72 & 65.89 & 65.83 & 64.82 & 61.98 & 60.22 \\
\hline 2 & 73.12 & 72.44 & 70.20 & 69.26 & 68.06 & 67.46 \\
\hline 3 & 82.20 & 81.17 & 80.98 & 80.09 & 77.34 & 76.69 \\
\hline 4 & 112.67 & 112.27 & 106.26 & 104.93 & 105.11 & 104.26 \\
\hline 5 & 115.98 & 114.90 & 110.62 & 109.76 & 108.71 & 108.02 \\
\hline 6 & 124.18 & 123.65 & 124.10 & 123.45 & 123.54 & 123.13 \\
\hline 7 & 129.35 & 128.39 & 124.90 & 123.95 & 127.59 & 126.77 \\
\hline 8 & 142.90 & 142.33 & 131.99 & 130.20 & 134.41 & 132.93 \\
\hline 9 & 145.65 & 147.60 & 146.08 & 144.14 & 140.94 & 139.80 \\
\hline 10 & 148.51 & 148.65 & 148.39 & 145.82 & 144.77 & 143.28 \\
\hline 20 & 193.18 & 192.46 & 198.62 & 196.93 & 190.12 & 188.69 \\
\hline 50 & 299.04 & 296.67 & 290.72 & 289.92 & 290.88 & 289.78 \\
\hline 100 & 410.74 & 408.81 & 404.63 & 403.36 & 401.95 & 400.77 \\
\hline
\end{tabular}
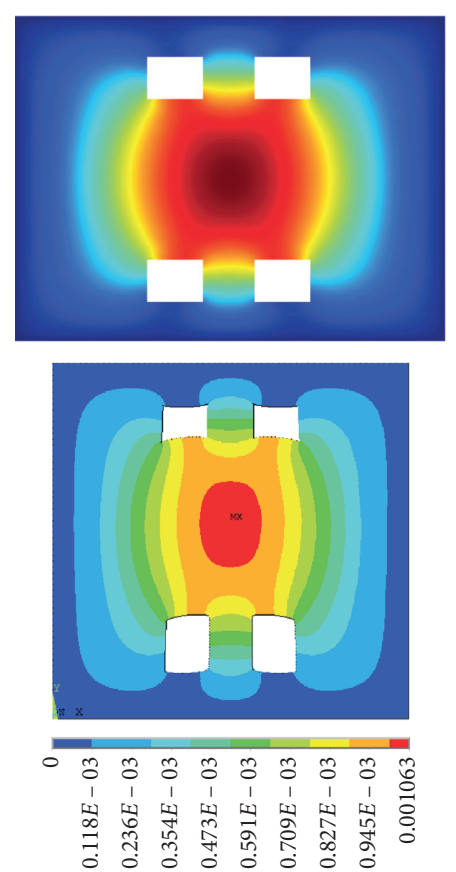

(a)
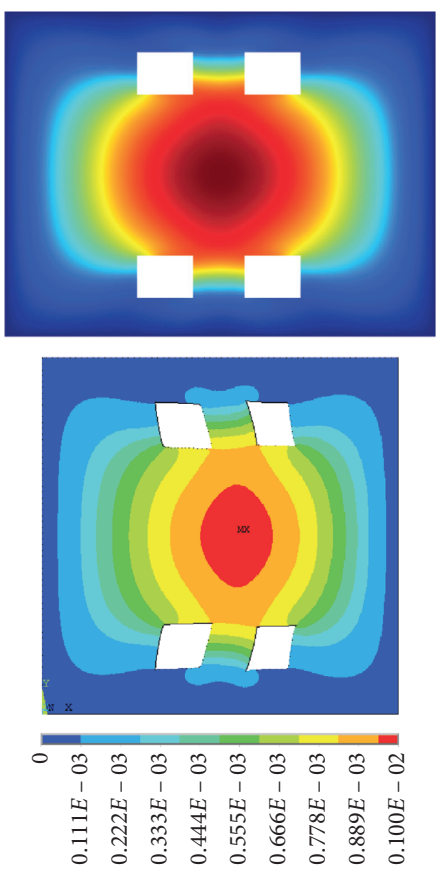

(b)
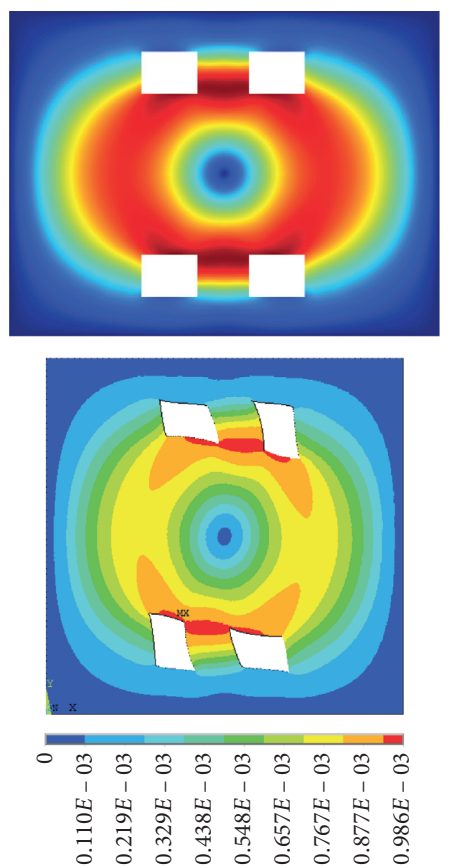

(c)

FIgURE 3: Continued. 

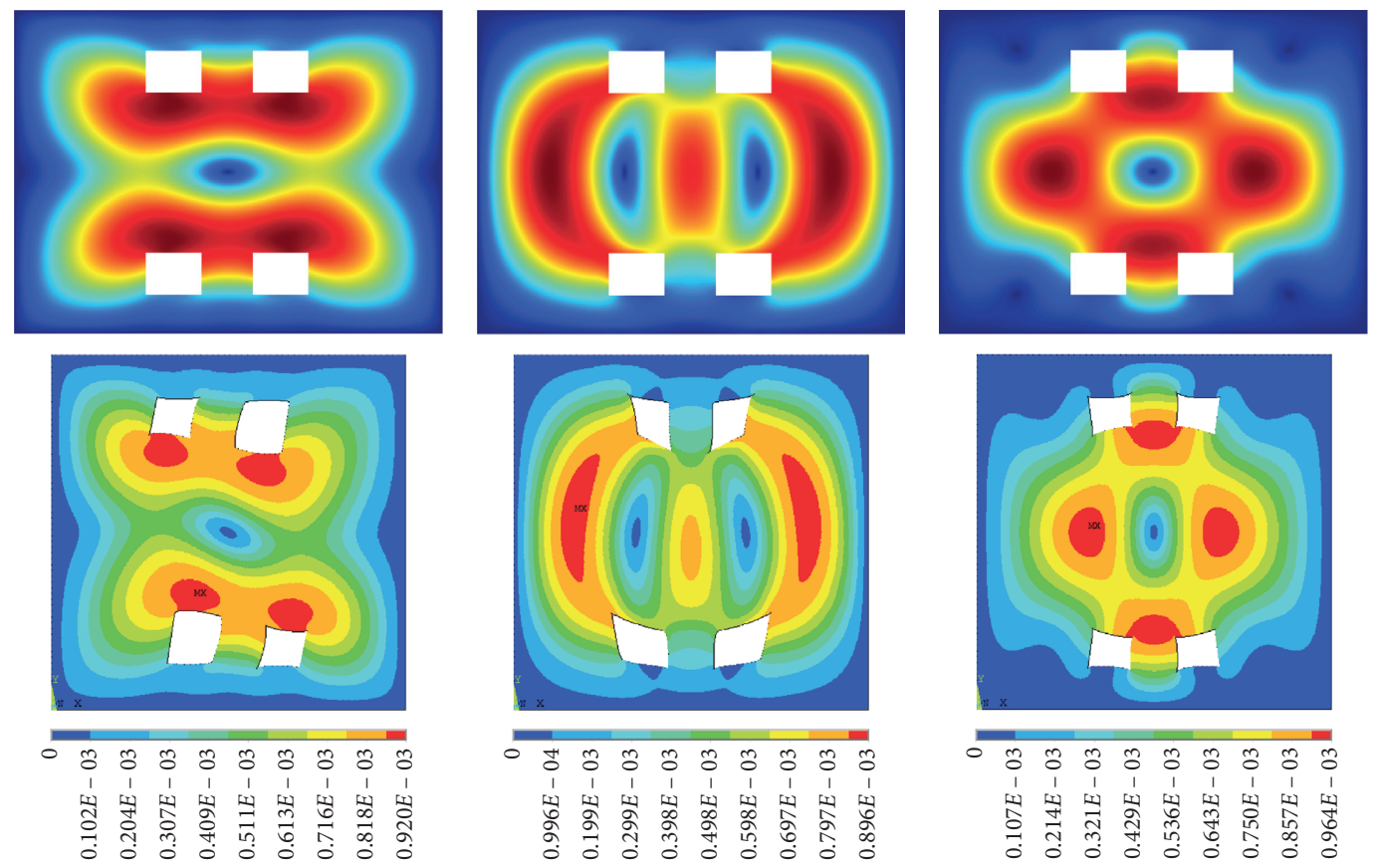

(e)

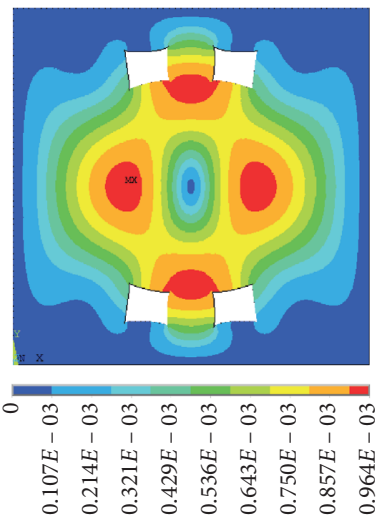

(f)

Figure 3: The first six mode shapes for the square plate with four cutouts. (a) The first mode; (b) the second mode; (c) the third mode; (d) the fourth mode; (e) the fifth mode; (f) the sixth mode. Upper: the present results; lower: the ANSYS results.
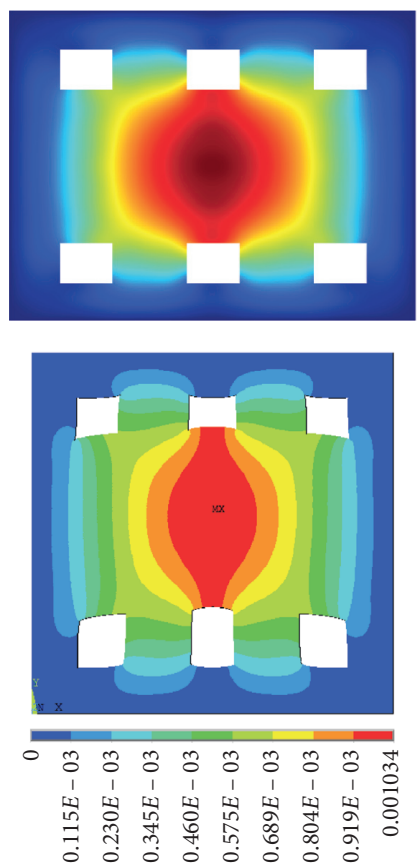

(a)
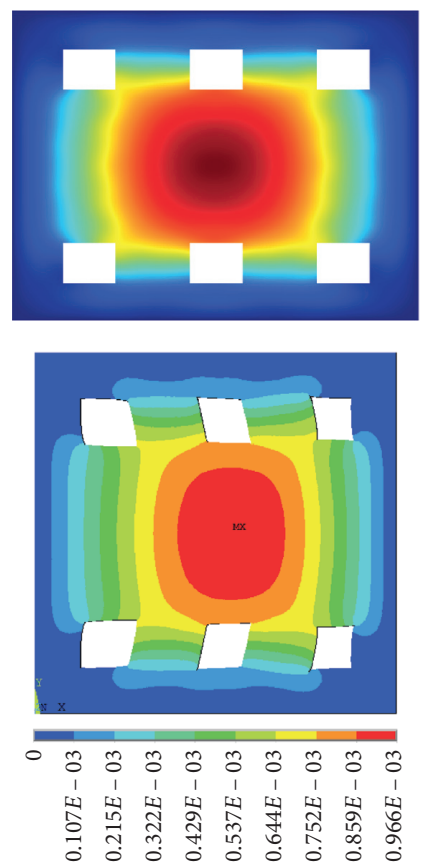

(b)
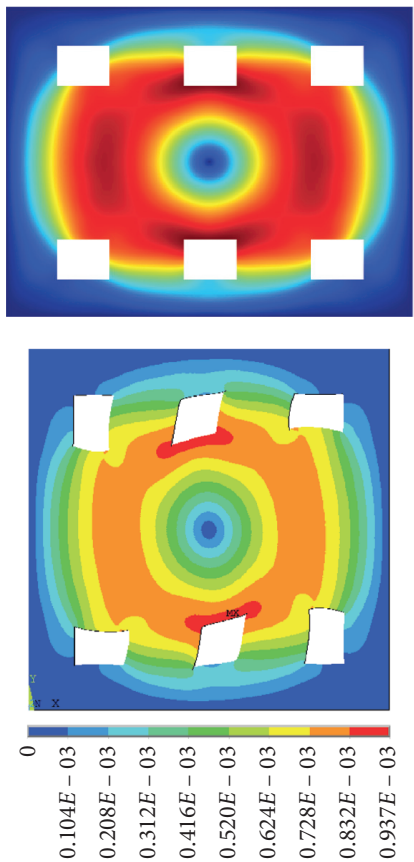

(c)

Figure 4: Continued. 

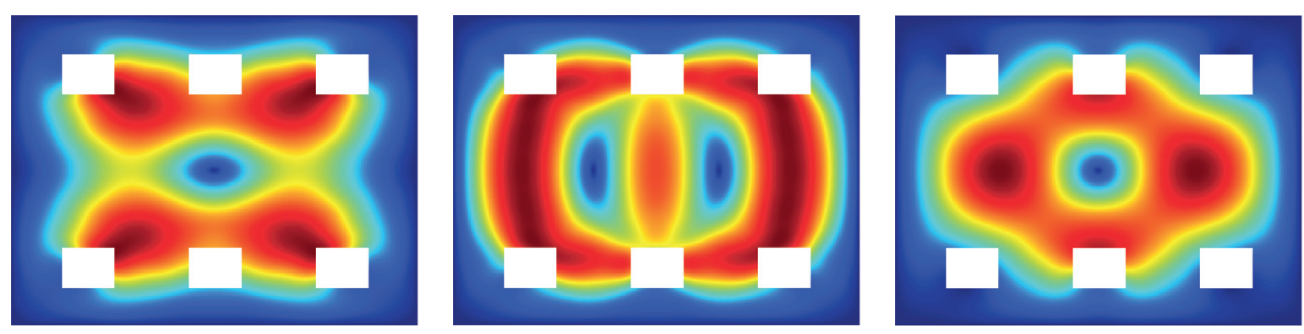

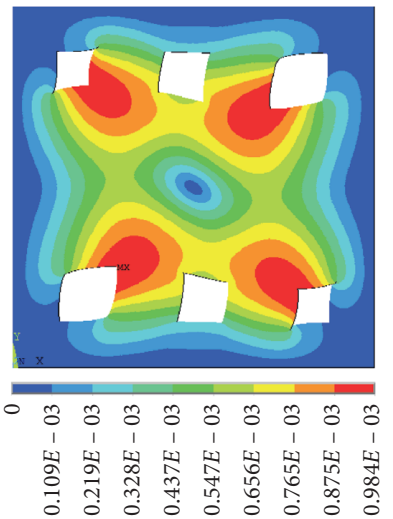

(d)

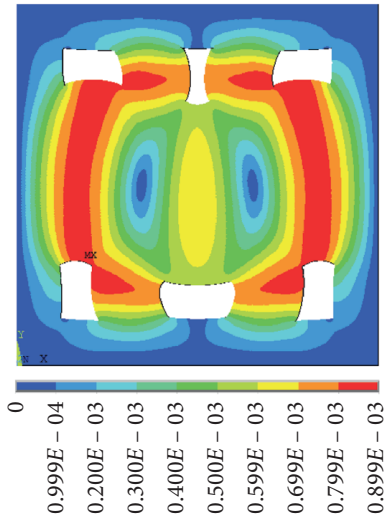

(e)

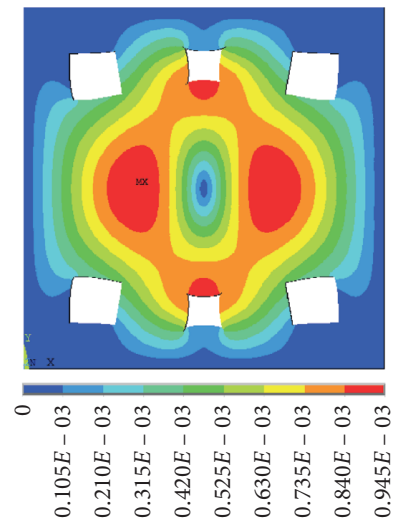

(f)

Figure 4: The first six mode shapes for the square plate with six cutouts. (a) The first mode; (b) the second mode; (c) the third mode; (d) the fourth mode; (e) the fifth mode; (f) the sixth mode. Upper: the present results; lower: the ANSYS results.
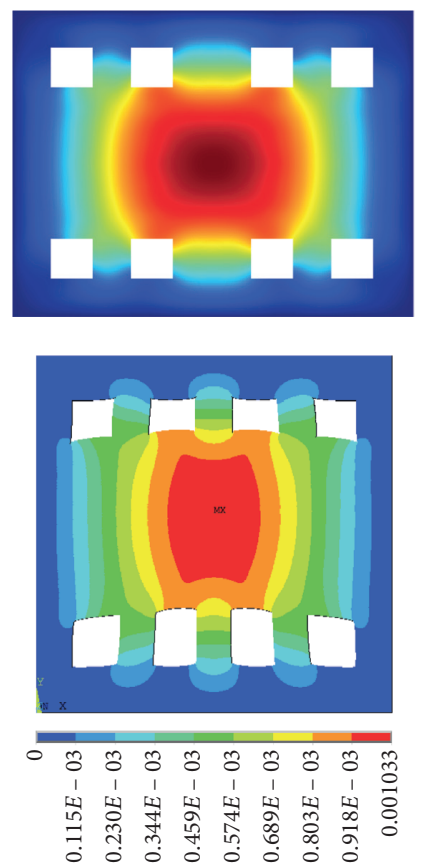

(a)
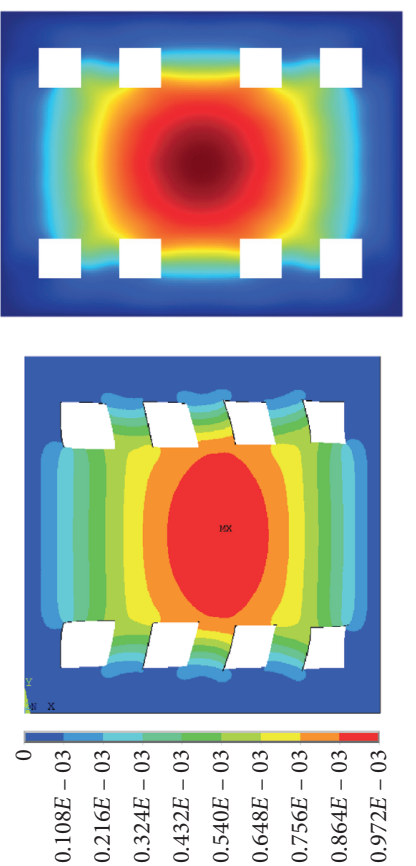

(b)
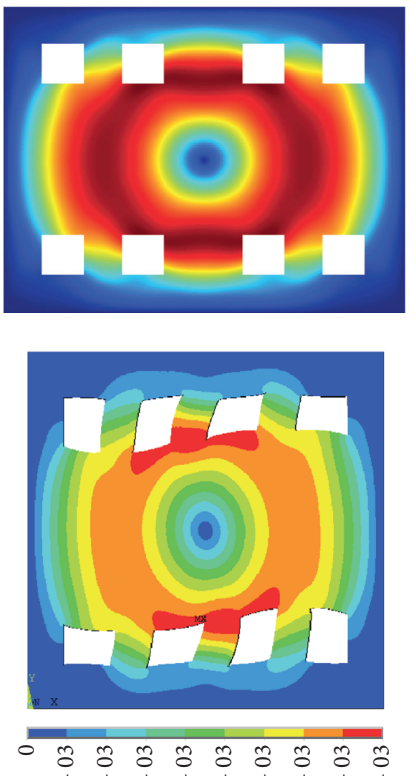

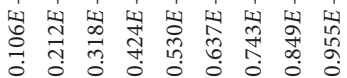

(c)
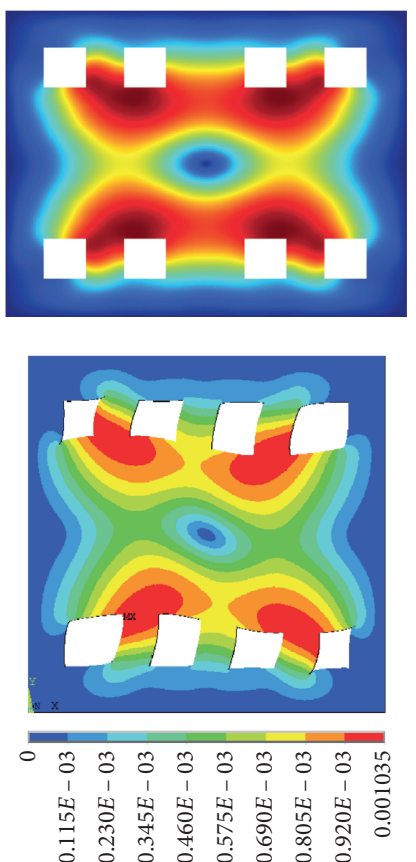

(d)

FIGURE 5: Continued. 

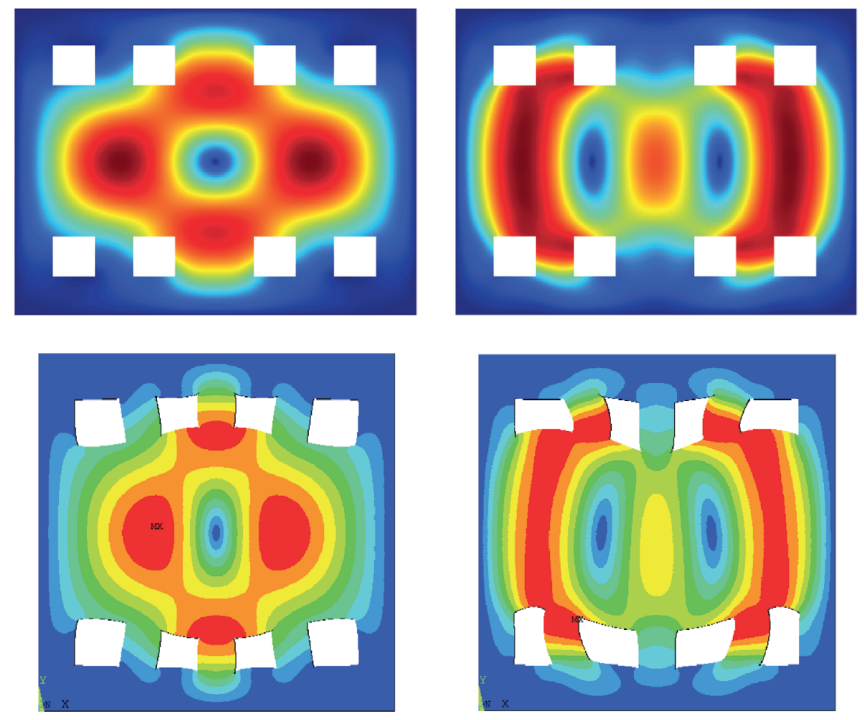

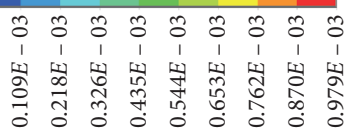

(f)

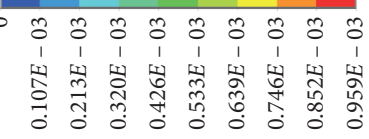

(e)

Figure 5: The first six mode shapes for the square plate with eight cutouts. (a) The first mode; (b) the second mode; (c) the third mode; (d) the fourth mode; (e) the fifth mode; (f) the sixth mode. Upper: the present results; lower: the ANSYS results.

TABLE 3: The natural frequencies of the rectangular plate with different cutout sizes.

\begin{tabular}{|c|c|c|c|c|c|c|}
\hline \multirow{2}{*}{ Mode number } & \multicolumn{2}{|c|}{$10 \mathrm{~m} \times 10 \mathrm{~m}$} & \multicolumn{2}{|c|}{$20 \mathrm{~m} \times 20 \mathrm{~m}$} & \multicolumn{2}{|c|}{$25 \mathrm{~m} \times 25 \mathrm{~m}$} \\
\hline & FEM & Present & FEM & Present & FEM & Present \\
\hline 1 & 70.61 & 70.95 & 83.29 & 83.54 & 103.38 & 103.82 \\
\hline 2 & 74.30 & 74.32 & 86.94 & 87.04 & 104.36 & 104.52 \\
\hline 3 & 85.96 & 86.13 & 97.35 & 97.61 & 136.02 & 136.08 \\
\hline 4 & 107.49 & 108.27 & 102.06 & 102.63 & 137.12 & 137.29 \\
\hline 5 & 115.16 & 115.42 & 120.23 & 120.34 & 144.29 & 144.35 \\
\hline 6 & 123.59 & 123.85 & 126.40 & 127.08 & 145.73 & 146.13 \\
\hline 7 & 128.91 & 128.94 & 141.30 & 141.50 & 152.01 & 152.43 \\
\hline 8 & 144.00 & 144.28 & 144.73 & 144.99 & 157.66 & 158.06 \\
\hline 9 & 149.48 & 149.73 & 158.55 & 158.72 & 164.98 & 165.07 \\
\hline 10 & 155.57 & 155.68 & 167.84 & 167.92 & 169.78 & 170.33 \\
\hline 20 & 209.85 & 210.01 & 226.07 & 226.36 & 241.77 & 241.95 \\
\hline 50 & 298.27 & 298.37 & 322.88 & 323.77 & 358.95 & 359.15 \\
\hline 100 & 416.65 & 416.77 & 460.46 & 460.95 & 499.36 & 500.90 \\
\hline
\end{tabular}

are shown in Table 3 and Figures 6-8, respectively. It can be seen from Table 3 that the comparisons of the natural frequencies for the three cases obtained by the present method and FEM are satisfactory. The phenomenon can be seen from Table 3 that the natural frequencies increase with the cutout size, which is attributed to the fact that the mass of the plate structure is decreased with the increase of cutout sizes. As shown in Figures 6-8, the mode shapes obtained by the present method are in good agreement with those obtained by FEM. The comparison of the mode shapes between three cases indicates that the mode shapes are influenced significantly by the cutout size.

To investigate the influence of the cutout positions on the modal characteristics, the natural frequencies and mode shapes for three plates with double cutouts having the same size are calculated. Three kinds of plates with cutouts are selected to investigate the influence of cutout positions. As shown in Figure 9, the coordinate positions of the origins for the double inner cutouts with $10 \mathrm{~m} \times 10 \mathrm{~m}$ size are $(5 \mathrm{~m}, 15 \mathrm{~m})$ and $(25 \mathrm{~m}, 15 \mathrm{~m})$, respectively. As shown in Figure 10, the coordinate positions of the origins for the double edge cutouts with $10 \mathrm{~m} \times 10 \mathrm{~m}$ size are $(15 \mathrm{~m}, 0 \mathrm{~m})$ and $(15 \mathrm{~m}, 30 \mathrm{~m})$, respectively. As shown in Figure 11, the coordinate positions of the origins for the double corner cutouts with $10 \mathrm{~m} \times 10 \mathrm{~m}$ size are $(0 \mathrm{~m}, 30 \mathrm{~m})$ and $(30 \mathrm{~m}, 30 \mathrm{~m})$, respectively. As shown in Table 4, the natural frequencies for the three cases from the present method and FEM are 

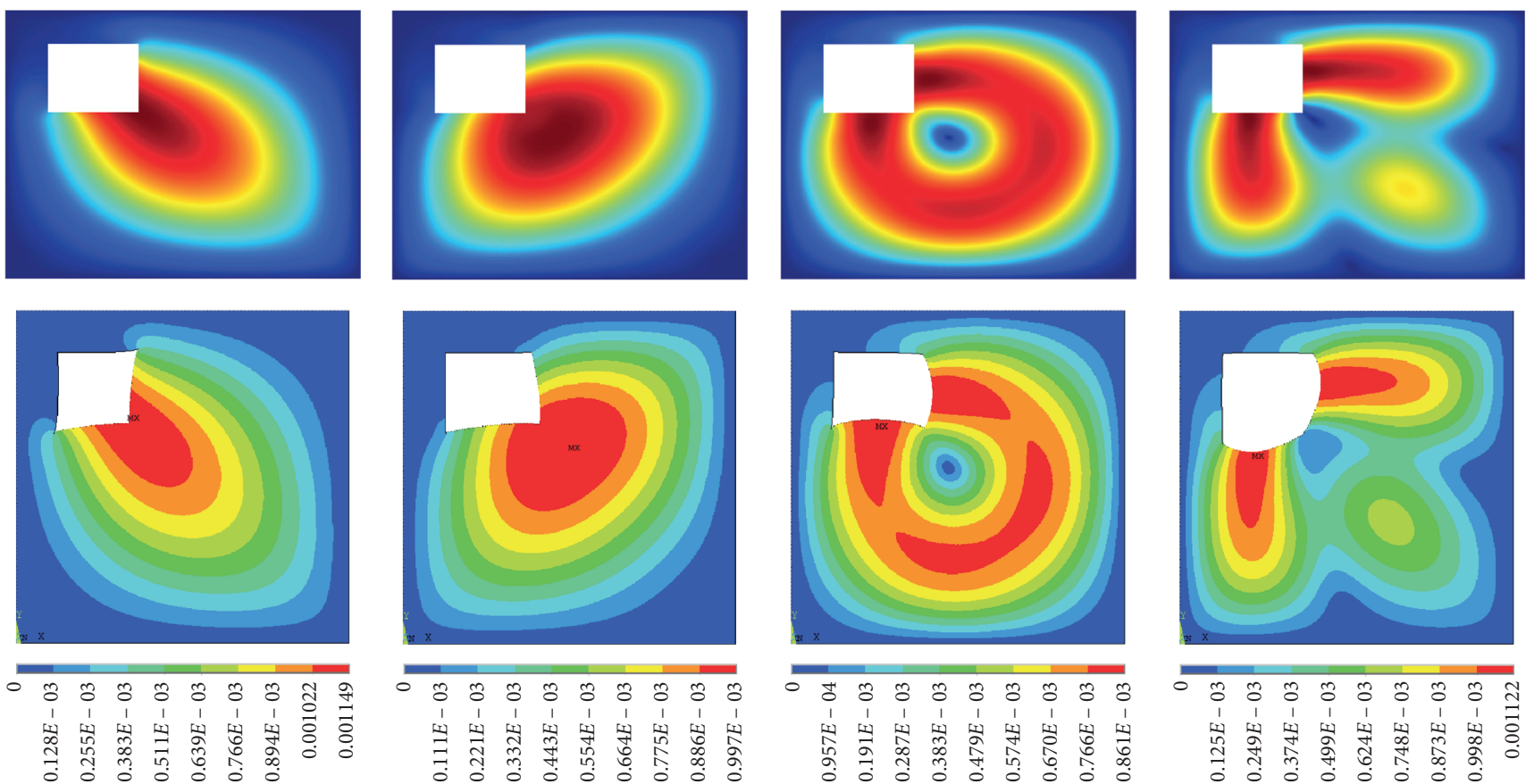

(a)

(b)
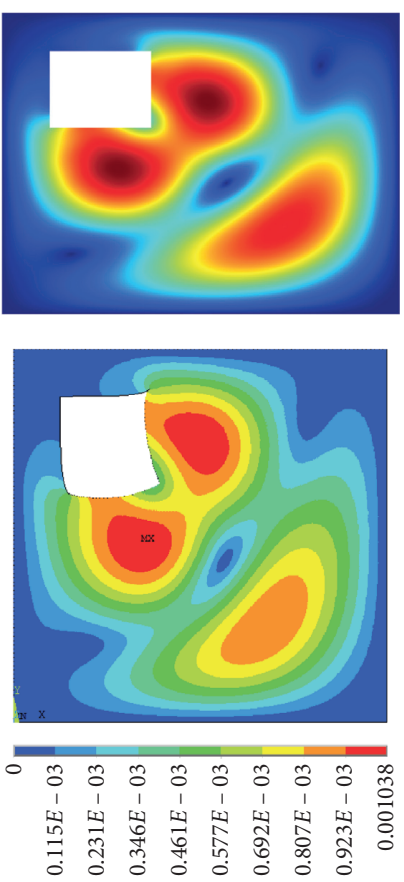

(f) (c)
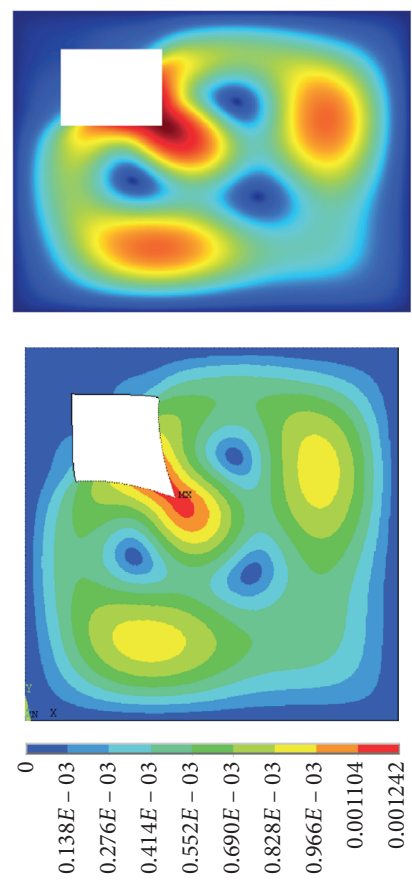

(e)

(d)

Figure 6: The first six mode shapes for the square plate with a cutout whose size is $10 \mathrm{~m} \times 10 \mathrm{~m}$. (a) The first mode; (b) the second mode; (c) the third mode; (d) the fourth mode; (e) the fifth mode; (f) the sixth mode. Upper: the present results; lower: the ANSYS results.

listed. We can see from Table 4 that the natural frequencies are in good agreement with those from the FEM and are greatly affected by the cutout positions. The first six mode shapes for the three cases from the present method and FEM are shown in Figures 9-11. An interesting phenomenon can be found that the distributions of the mode shapes are dependent on the cutout positions by comparing Figures 9-11. 

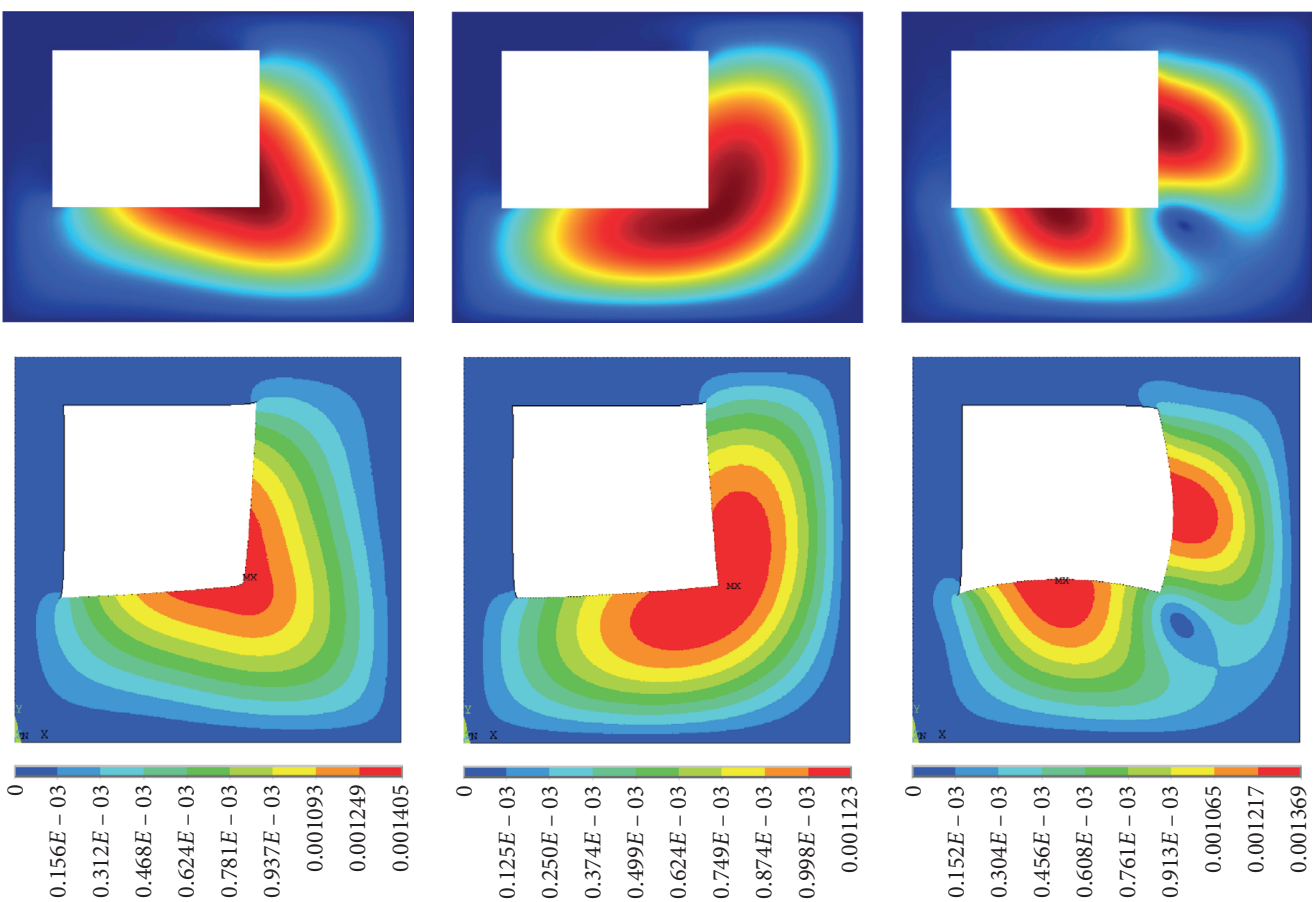

(a)

(b)
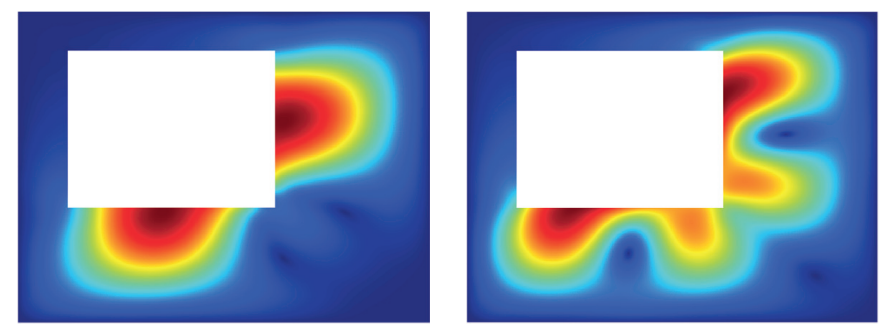

(c)
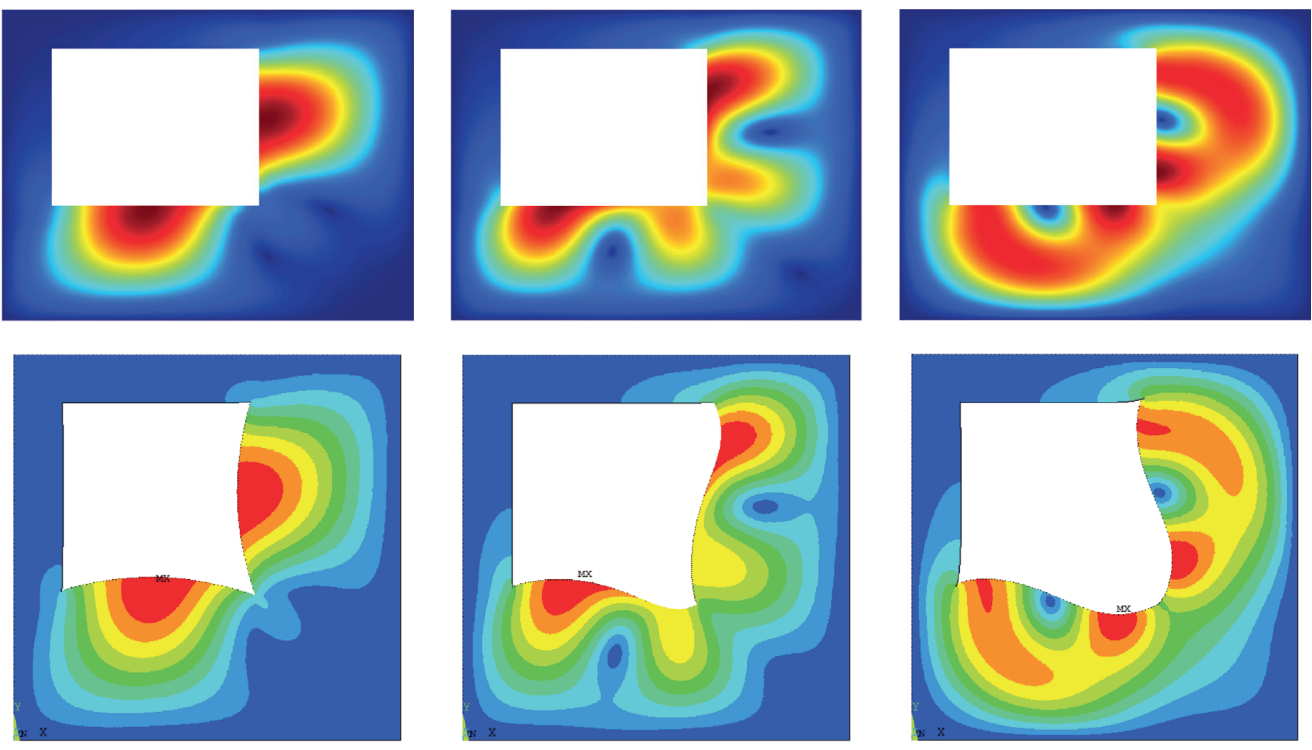

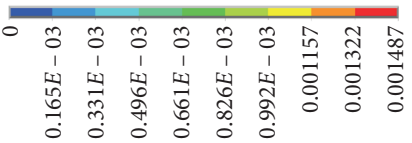

(d)

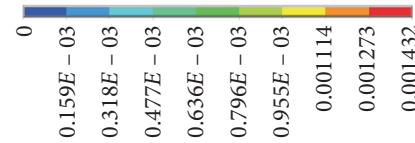

(e)

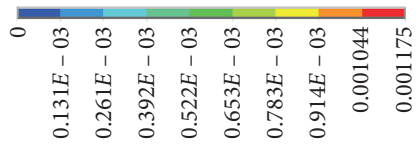

(f)

FIgURE 7: The first six mode shapes for the square plate with a cutout whose size is $20 \mathrm{~m} \times 20 \mathrm{~m}$. (a) The first mode; (b) the second mode; (c) the third mode; (d) the fourth mode; (e) the fifth mode; (f) the sixth mode. Upper: the present results; lower: the ANSYS results. 

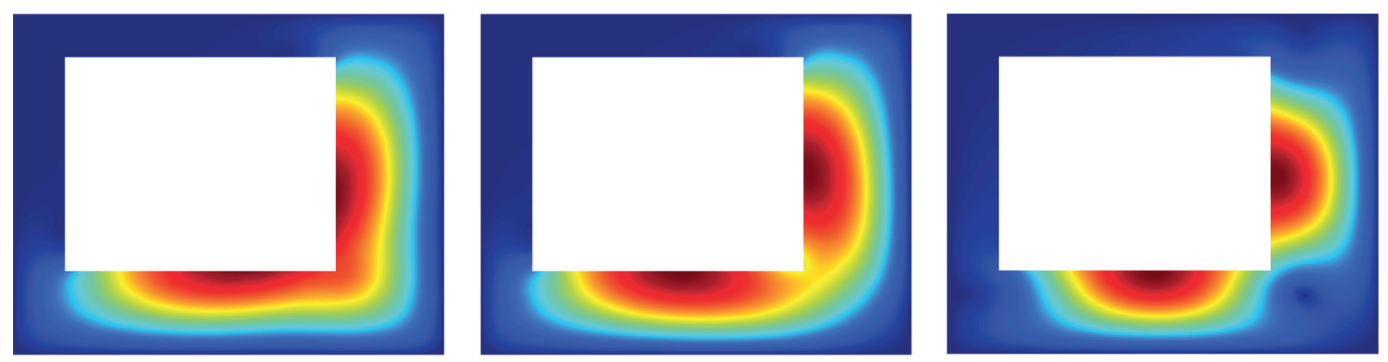

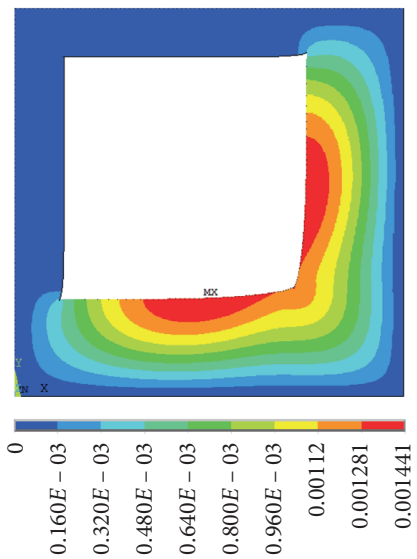

(a)
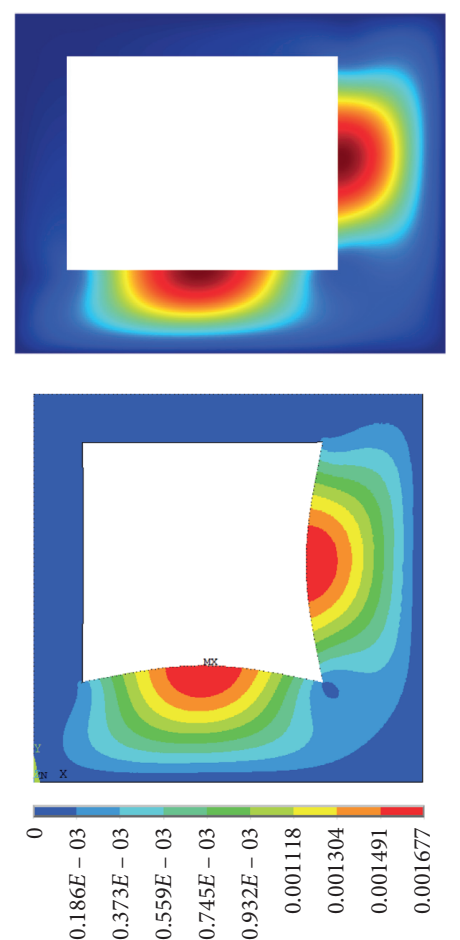

(d)

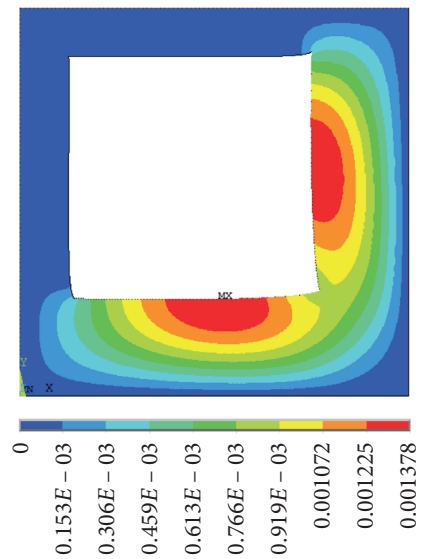

(b)
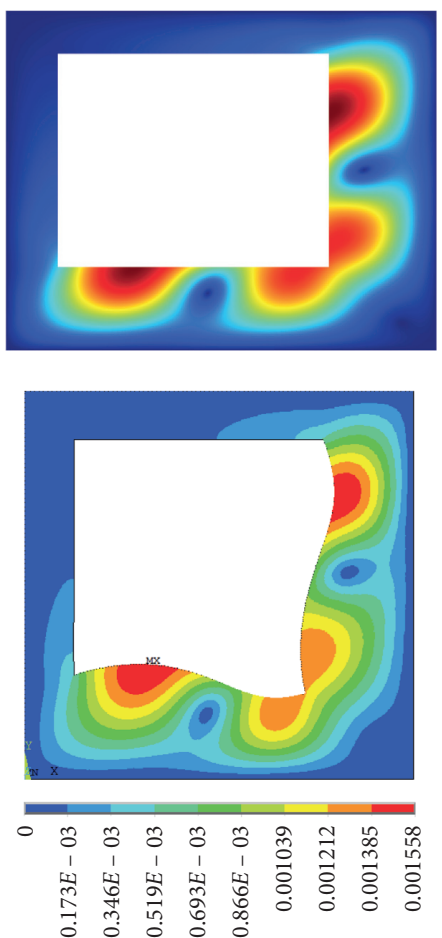

(e)

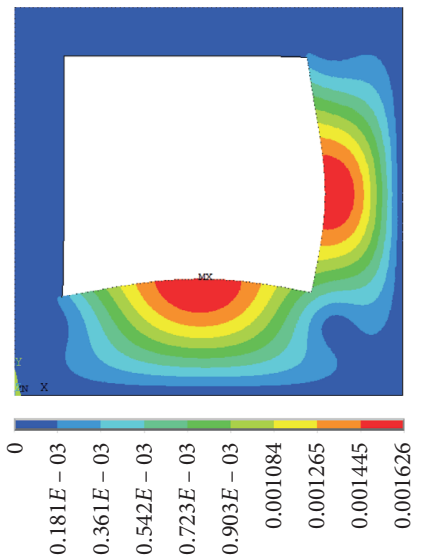

(c)
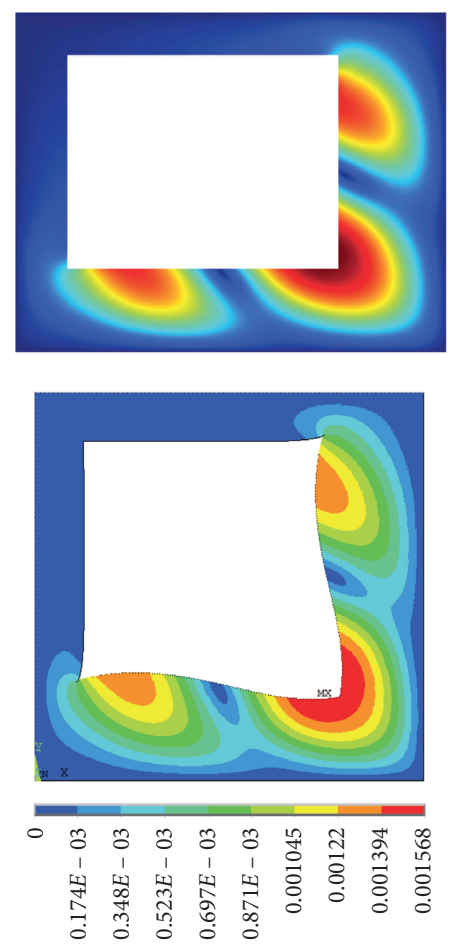

(f)

Figure 8: The first six mode shapes for the square plate with a cutout whose size is $25 \mathrm{~m} \times 25 \mathrm{~m}$. (a) The first mode; (b) the second mode; (c) the third mode; (d) the fourth mode; (e) the fifth mode; (f) the sixth mode. Upper: the present results; lower: the ANSYS results. 

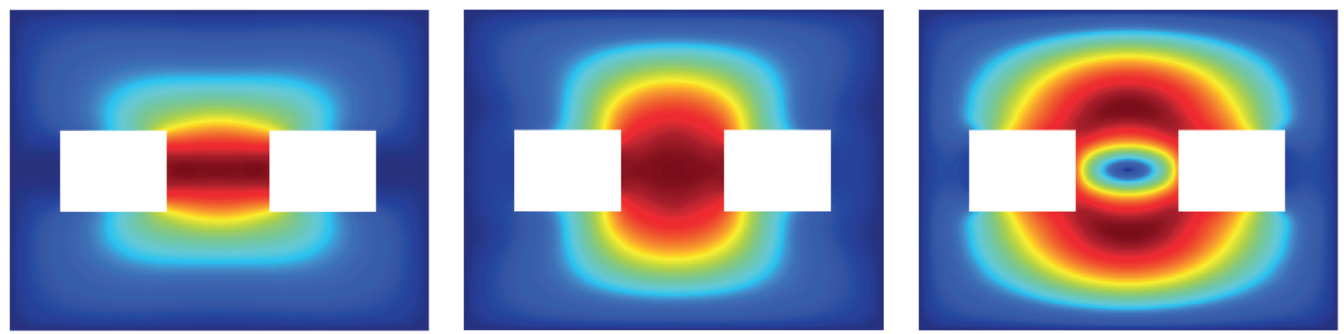

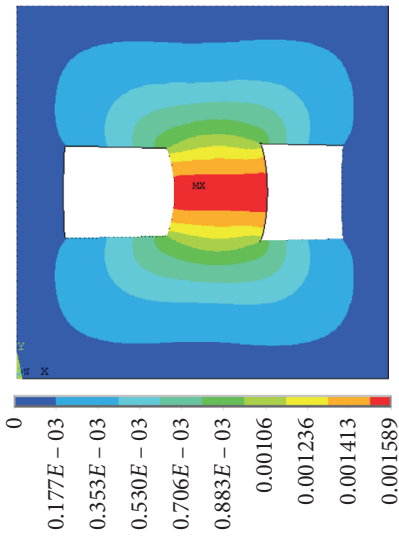

(a)
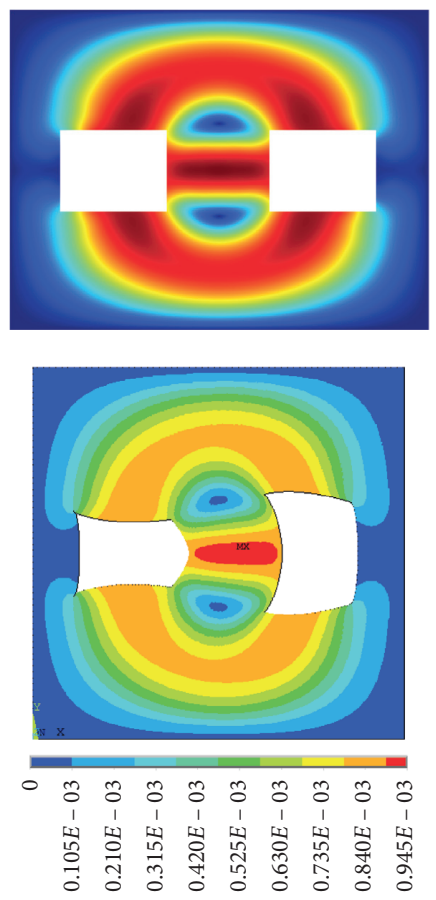

(d)

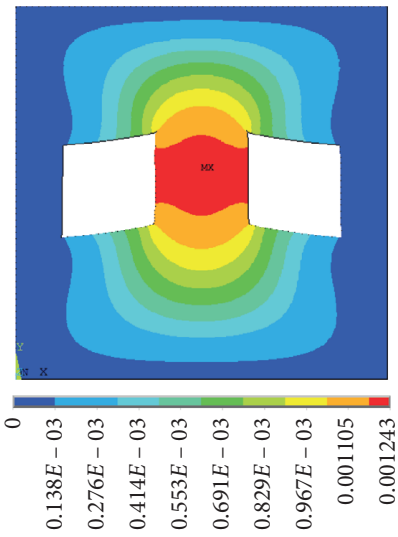

(b)
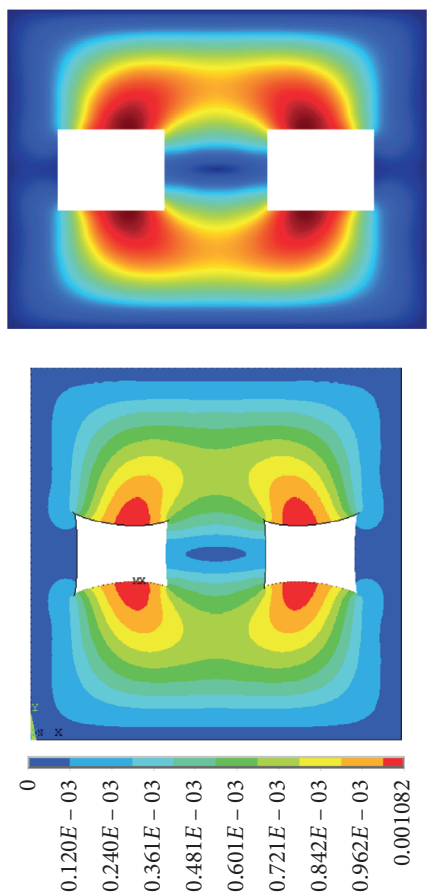

(e)

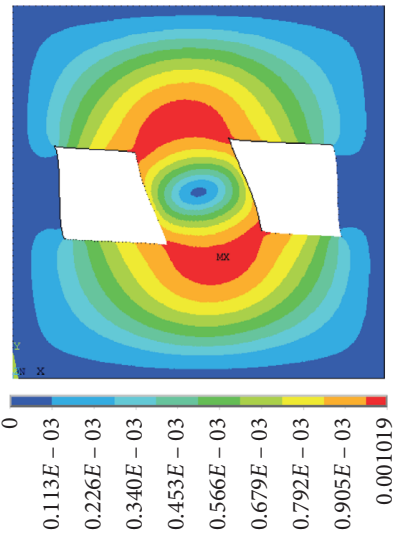

(c)
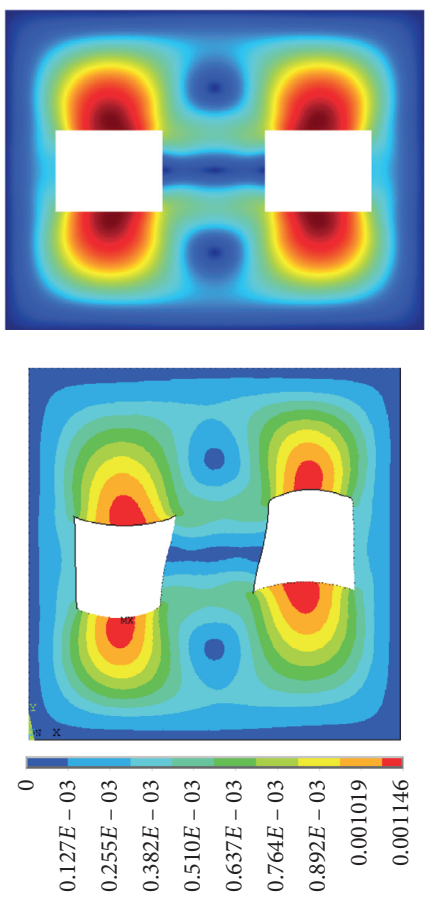

(f)

Figure 9: The first six mode shapes for the square plate with double inner cutouts. (a) The first mode; (b) the second mode; (c) the third mode; (d) the fourth mode; (e) the fifth mode; (f) the sixth mode. Upper: the present results; lower: the ANSYS results. 

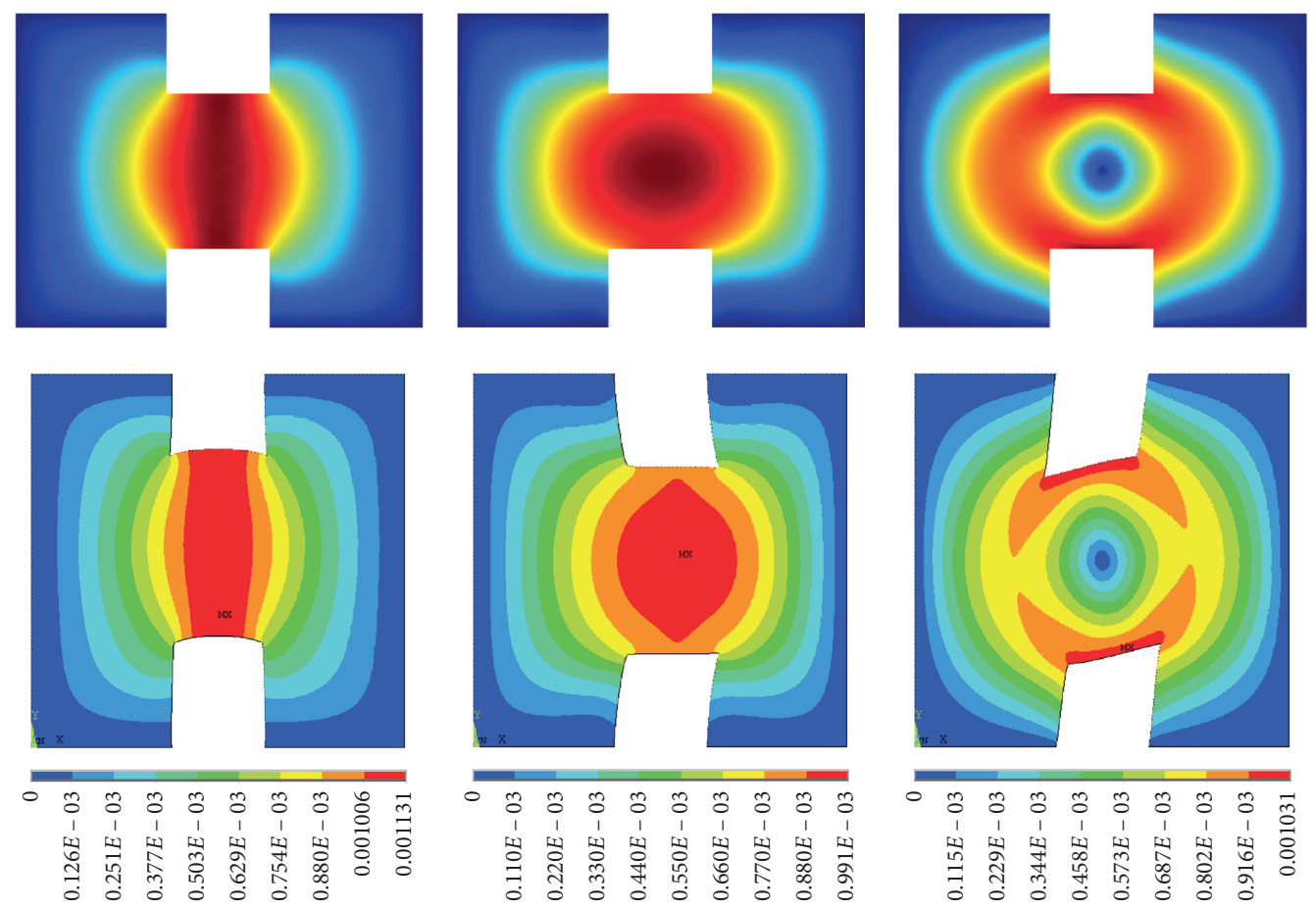

(a)

(b)
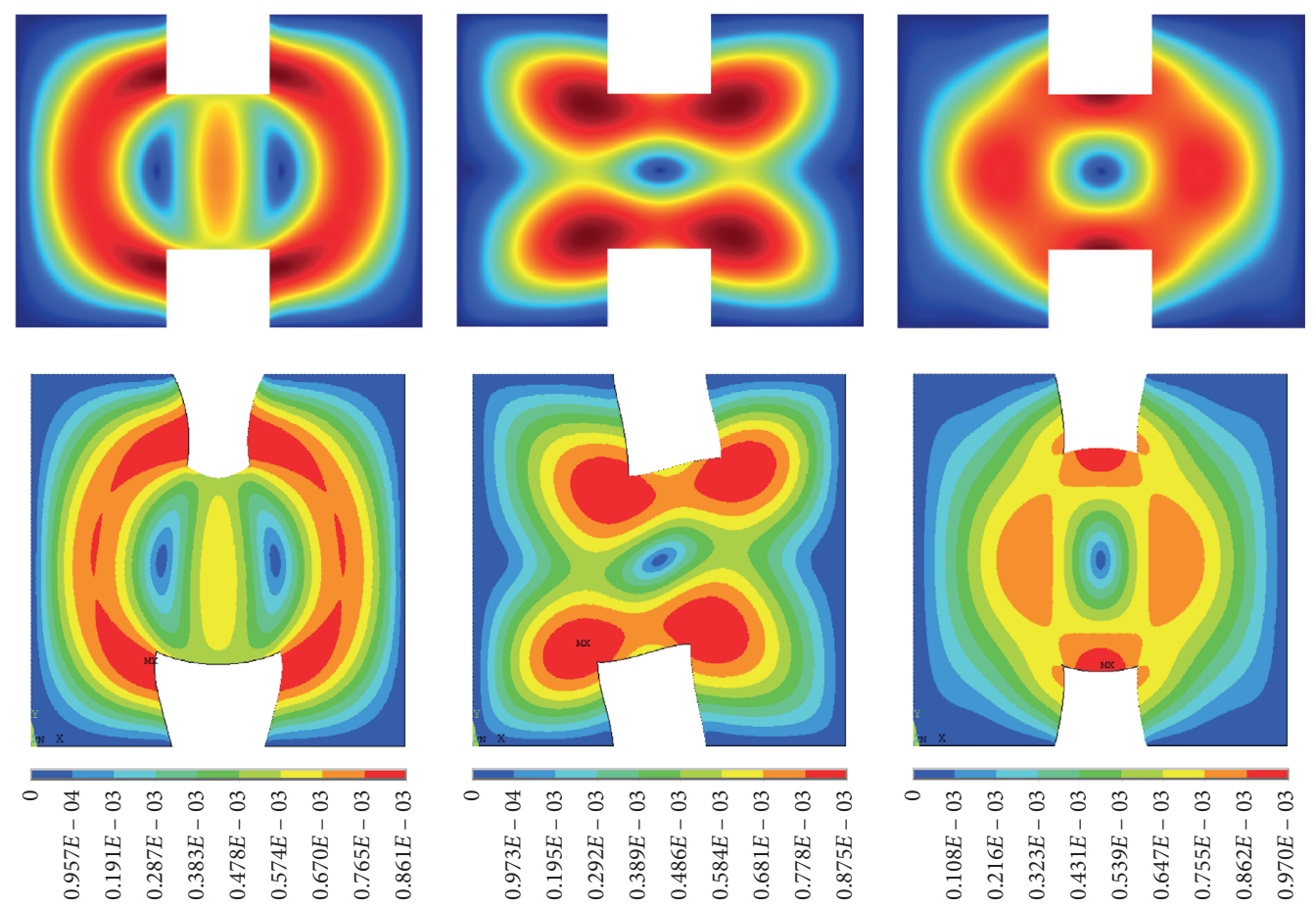

(d)

(e)

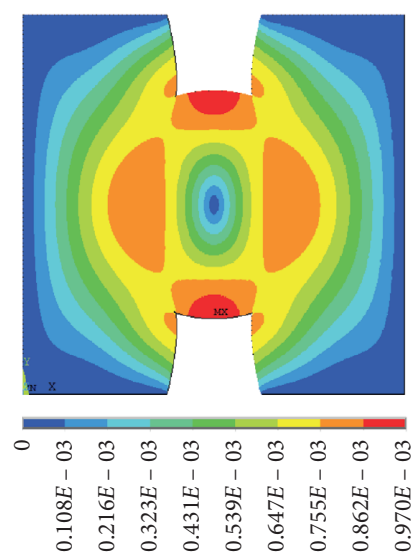

(f)

Figure 10: The first six mode shapes for the square plate with double edge cutouts. (a) The first mode; (b) the second mode; (c) the third mode; (d) the fourth mode; (e) the fifth mode; (f) the sixth mode. Upper: the present results; lower: the ANSYS results. 

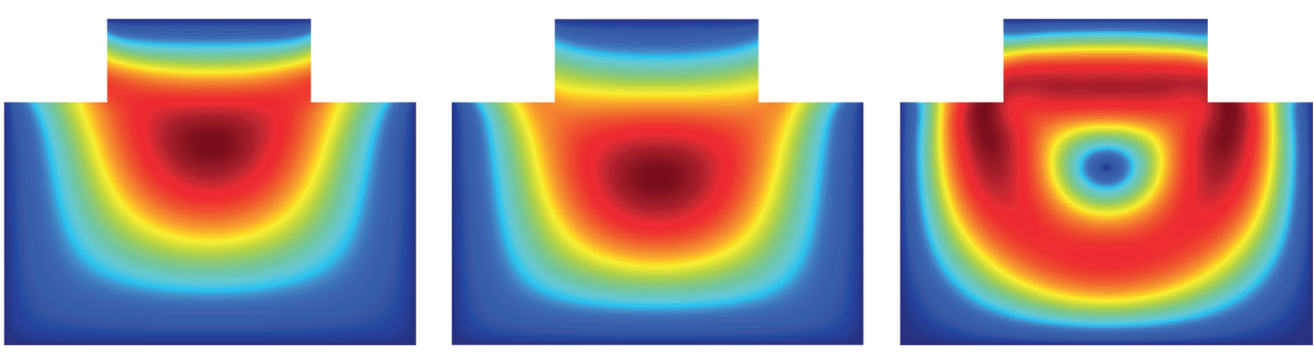
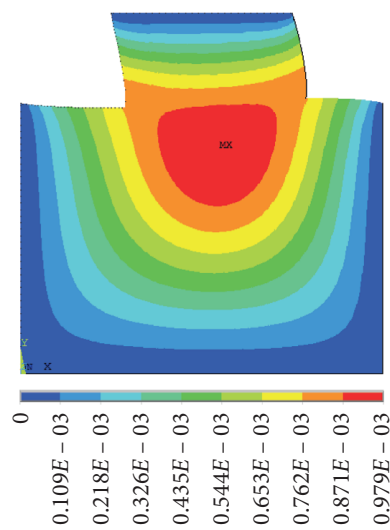

(a)
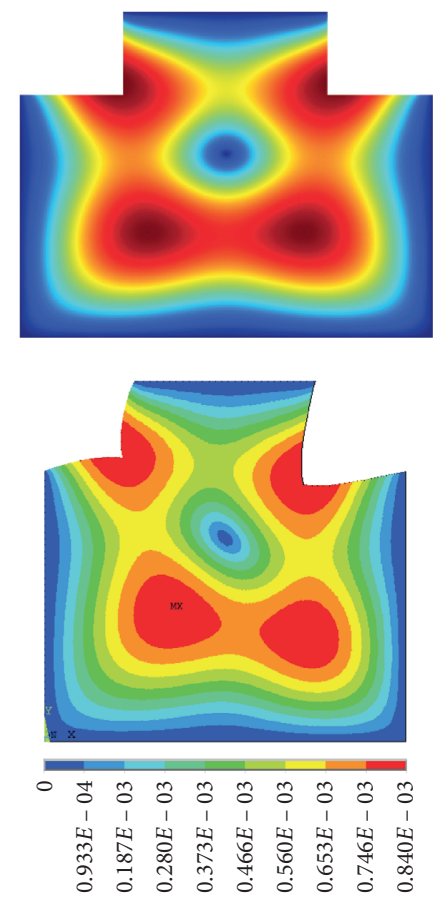

(d)

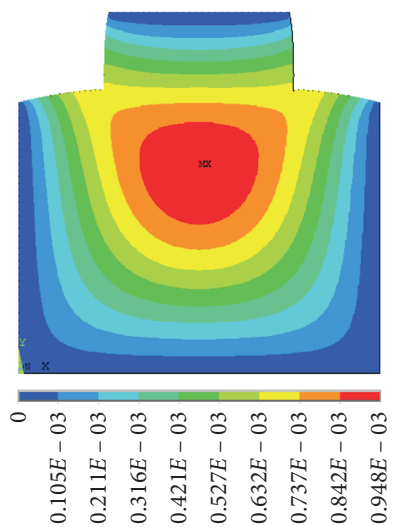

(b)
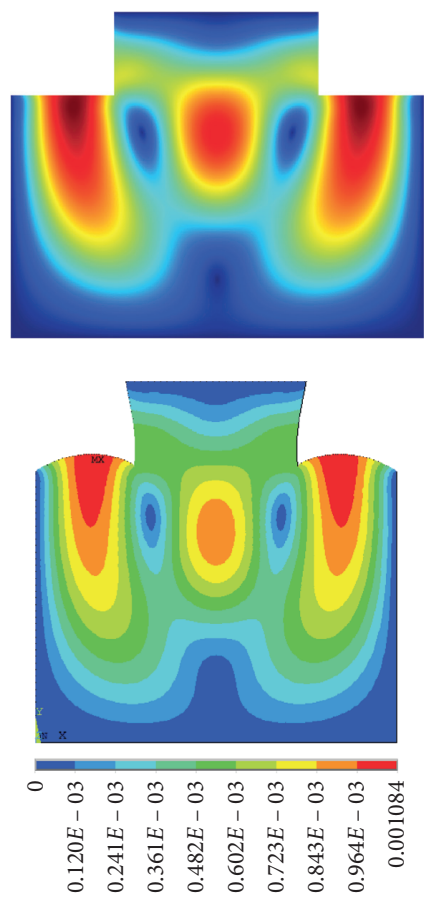

(e)

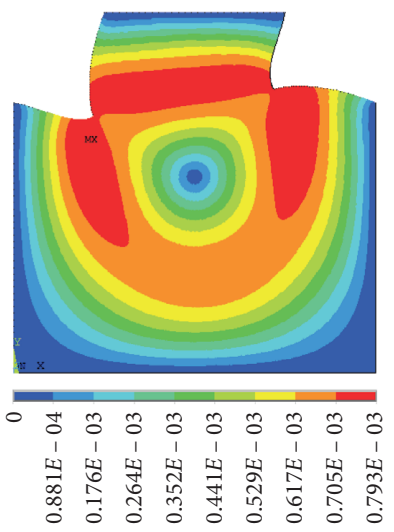

(c)
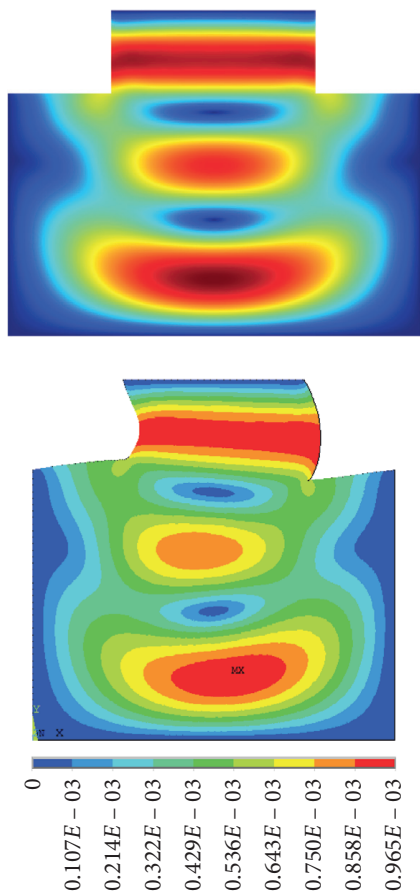

(f)

Figure 11: The first six mode shapes for the square plate with double corner cutouts. (a) The first mode; (b) the second mode; (c) the third mode; (d) the fourth mode; (e) the fifth mode; (f) the sixth mode. Upper: the present results; lower: the ANSYS results. 
TABLE 4: The natural frequencies of the rectangular plates for different cutout positions.

\begin{tabular}{|c|c|c|c|c|c|c|}
\hline \multirow{2}{*}{ Mode number } & \multicolumn{2}{|c|}{ Inner cutouts } & \multicolumn{2}{|c|}{ Edge cutouts } & \multicolumn{2}{|c|}{ Corner cutouts } \\
\hline & ANSYS & Present & ANSYS & Present & ANSYS & Present \\
\hline 1 & 65.55 & 66.18 & 60.83 & 61.26 & 69.10 & 69.12 \\
\hline 2 & 77.36 & 77.78 & 73.13 & 73.39 & 70.24 & 70.25 \\
\hline 3 & 83.79 & 84.31 & 80.68 & 80.94 & 82.97 & 83.00 \\
\hline 4 & 103.43 & 104.18 & 109.81 & 109.89 & 102.56 & 102.60 \\
\hline 5 & 113.76 & 114.51 & 113.00 & 113.01 & 117.27 & 117.42 \\
\hline 6 & 114.00 & 114.60 & 119.77 & 119.97 & 118.67 & 118.87 \\
\hline 7 & 127.32 & 128.43 & 127.25 & 127.27 & 123.85 & 123.87 \\
\hline 8 & 130.58 & 133.21 & 129.20 & 129.25 & 138.92 & 138.93 \\
\hline 9 & 138.36 & 139.06 & 141.20 & 141.96 & 148.05 & 148.16 \\
\hline 10 & 140.72 & 141.39 & 142.41 & 143.49 & 148.29 & 148.30 \\
\hline 20 & 197.79 & 198.16 & 199.63 & 199.66 & 204.38 & 204.51 \\
\hline 50 & 297.65 & 298.65 & 300.66 & 301.29 & 307.39 & 308.11 \\
\hline 100 & 424.80 & 425.83 & 426.14 & 426.87 & 423.74 & 424.11 \\
\hline
\end{tabular}

\section{Conclusion}

The energy principle combined with the Rayleigh-Ritz method has been adopted to predict the in-plane modal characteristics of the plate with cutouts, in which the number, size, and position of cutouts are variable. In the theoretical formulation, the cutouts within the structure are considered by subtracting the energy occupied by the cutouts area from the total energy of the whole plate. The inplane displacements of the plate with cutouts are expressed as improved Fourier series to ensure the smoothness and continuity over the entire solution domain. The convergence of the present method is checked by calculating the natural frequencies for the square plate with an inner cutout under different truncation series. The accuracies of the modal results of all the numerical examples considered in the Results and Discussion have been validated by the comparisons with those obtained from FEM. By investigating the influence of the number of the cutouts on the in-plane modal characteristics of the square plate, we can find that the increase of the number of cutouts will decrease the structural stiffness and the natural frequencies. The reason why the square plate has a larger cutout with higher natural frequencies is attributed to the fact that the structure mass decreased with the increase of the cutout sizes. It can be found that the distributions of mode shapes are influenced by the cutout positions by investigating the positions of the multiple cutouts.

\section{Appendix}

The representative expressions of the first row of submatrices in the matrices $K_{\mathrm{i}}, M_{\mathrm{i}}, K$, and $M$ are given in this section. For the convenience of description, we will introduce some indices listed in the following:

$$
\begin{aligned}
S_{1} & =m_{1}(N+1)+n_{1}+1, \\
T_{1} & =m_{2}(N+1)+n_{2}+1, \\
T_{2} & =m_{2}+1, \\
T_{3} & =m_{2}+1, \\
T_{4} & =n_{2}+1, \\
T_{5} & =n_{2}+1, \\
T_{6} & =m_{2}(N+1)+n_{2}+1, \\
T_{7} & =m_{2}+1, \\
T_{8} & =m_{2}+1, \\
T_{9} & =m_{2}+1, \\
T_{10} & =n_{2}+1 .
\end{aligned}
$$

Taking the derivatives of the Lagrangian energy functions with respect to the undetermined coefficients $B_{m n}$ and making them equal to zero, the submatrices in the first row of $K_{\mathrm{i}}$ can be obtained as 


$$
\begin{aligned}
\left\{K_{1-1}^{i}\right\}_{S_{1}, T_{1}}= & \lambda_{a m 1} \lambda_{a m 2}\left(\int_{X c i}^{X d i} \sin \lambda_{a m 1} x \sin \lambda_{a m 2} x \mathrm{~d} x\right)\left(\int_{Y c i}^{Y d i} \cos \lambda_{b n 1} y \cos \lambda_{b n 2} y \mathrm{~d} y\right) \\
& +\frac{1-u_{1}}{2}\left(\lambda_{b n 1} \lambda_{b n 2}\left(\int_{X c i}^{X d i} \cos \lambda_{a m 1} x \cos \lambda_{a m 2} x \mathrm{~d} x\right)\left(\int_{Y c i}^{Y d i} \sin \lambda_{b n 1} y \sin \lambda_{b n 2} y \mathrm{~d} y\right)\right) \\
& +k_{n x 0} \int_{Y c i}^{Y d i} \cos \lambda_{b n 1} y \cos \lambda_{b n 2} y \mathrm{~d} y+k_{n x 1}(-1)^{m_{1}+m_{2}} \int_{Y c i}^{Y d i} \cos \lambda_{b n 1} y \cos \lambda_{b n 2} y \mathrm{~d} y \\
& +k_{p y 0} \int_{X c i}^{X d i} \cos \lambda_{a m 1} x \cos \lambda_{a m 2} x \mathrm{~d} x+k_{p y 1}(-1)^{n_{1}+n_{2}} \int_{X c i}^{X d i} \cos \lambda_{a m 1} x \cos \lambda_{a m 2} x \mathrm{~d} x, \\
\left\{K_{1-2}^{i}\right\}_{S_{1}, T_{2}} & \lambda_{a m 1} \lambda_{a m 2}\left(\int_{X c i}^{X d i} \sin \lambda_{a m 1} x \sin \lambda_{a m 2} x \mathrm{~d} x\right)\left(\int_{Y c i}^{Y d i} \xi_{1 b}(y) \cos \lambda_{b n 1} y \mathrm{~d} y\right) \\
& +\frac{1-u_{1}\left(-\lambda_{b n 1} \int_{X c i}^{X d i} \cos \lambda_{a m 1} x \cos \lambda_{a m 2} x \mathrm{~d} x \int_{Y c i}^{Y d i} \xi_{1 b}^{\prime}(y) \sin \lambda_{b n 1} y \mathrm{~d} y\right)}{2} \\
& +k_{n x 0} \int_{Y c i}^{Y d i} \xi_{1 b}(y) \cos \lambda_{b n 1} y \mathrm{~d} y+k_{n x 1}(-1)^{m_{1}+m_{2}} \int_{Y c i}^{Y d i} \xi_{1 b} \cos \lambda_{b n 1} y \mathrm{~d} y .
\end{aligned}
$$

The submatrices $K_{1-3}^{i}$ can be derived by replacing $\xi_{1 b}(y)$ in submatrices $K_{1-2}^{i}$ with $\xi_{2 b}(y)$.

$$
\begin{aligned}
\left\{K_{1-4}^{i}\right\}_{S_{1}, T_{4}}= & -\lambda_{a m 1} \int_{X c i}^{X d i} \xi_{1 a}^{\prime}(x) \sin \lambda_{a m 1} x \mathrm{~d} x \int_{Y c i}^{Y d i} \cos \lambda_{b n 1} y \cos \lambda_{b n 2} y \mathrm{~d} y \\
& +\frac{1-u_{1}}{2}\left(\lambda_{b n 1} \lambda_{b n 2} \int_{X c i}^{X d i} \xi_{1 a}(x) \cos \lambda_{a m 1} x \mathrm{~d} x \int_{Y c i}^{Y d i} \sin \lambda_{b n 1} x \sin \lambda_{b n 2} x \mathrm{~d} x\right) \\
& +k_{p y 0} \int_{X c i}^{X d i} \xi_{1 a}(x) \cos \lambda_{a m 1} x \mathrm{~d} x+k_{p y 1}(-1)^{n_{1}+n_{2}} \int_{X c i}^{X d i} \xi_{1 a}(x) \cos \lambda_{a m 1} x \mathrm{~d} x .
\end{aligned}
$$

The submatrices $K_{1-5}^{i}$ can be derived by replacing $\xi_{1 a}(x)$ in submatrices $K_{1-4}^{i}$ with $\xi_{2 a}(x)$.

$$
\begin{aligned}
\left\{K_{1-6}^{i}\right\}_{S_{1}, T_{6}}= & u_{1}\left(\lambda_{b n 2} \lambda_{a m 1} \int_{X c i}^{X d i} \sin \lambda_{a m 1} x \cos \lambda_{a m 2} x \mathrm{~d} x \int_{Y c i}^{Y d i} \cos \lambda_{b n 1} y \sin \lambda_{b n 2} y \mathrm{~d} y\right) \\
& +\frac{1-u_{1}}{2}\left(\lambda_{b n 1} \lambda_{a m 2} \int_{X c i}^{X d i} \cos \lambda_{a m 1} x \sin \lambda_{a m 2} x \mathrm{~d} x \int_{Y c i}^{Y d i} \sin \lambda_{b n 1} y \cos \lambda_{b n 2} y \mathrm{~d} y\right) \\
\left\{K_{1-7}^{i}\right\}_{S_{1}, T_{7}}= & u_{1}\left(-\lambda_{a m 1}\right) \int_{X c i}^{X d i} \sin \lambda_{a m 1} x \cos \lambda_{a m 2} x \mathrm{~d} x \int_{Y c i}^{Y d i} \xi_{1 b}^{\prime}(y) \cos \lambda_{b n 1} y \mathrm{~d} y \\
& +\frac{1-u_{1}}{2}\left(\lambda_{b n 1} \lambda_{b n 2} \int_{X c i}^{X d i} \cos \lambda_{a m 1} x \sin \lambda_{a m 2} x \mathrm{~d} x \int_{Y c i}^{Y d i} \xi_{1 b}(y) \sin \lambda_{b n 1} y \mathrm{~d} y\right)
\end{aligned}
$$

The submatrices $K_{1-8}^{i}$ can be derived by replacing $\xi_{1 b}(y)$ in submatrices $K_{1-7}^{i}$ with $\xi_{2 b}(y)$. 


$$
\begin{aligned}
\left\{K_{1-9}^{i}\right\}_{S_{1}, T_{9}}= & u_{1}\left(\lambda_{b n 2} \lambda_{a m 1} \int_{X c i}^{X d i} \xi_{1 a}(x) \sin \lambda_{a m 1} x \mathrm{~d} x \int_{Y c i}^{Y d i} \cos \lambda_{b n 1} y \sin \lambda_{b n 2} y \mathrm{~d} y\right) \\
& +\frac{1-u_{1}}{2}\left(\left(-\lambda_{b n 1}\right) \int_{X c i}^{X d i} \xi_{1 a}(x) \cos \lambda_{a m 1} x \mathrm{~d} x \int_{Y c i}^{Y d i} \sin \lambda_{b n 1} y \cos \lambda_{b n 2} y \mathrm{~d} y\right) .
\end{aligned}
$$

The submatrices $K_{1-10}^{i}$ can be derived by replacing $\xi_{1 a}(x)$ in submatrices $K_{1-9}^{i}$ with $\xi_{2 a}(x)$.

Taking the derivatives of the Lagrangian energy functions with respect to the undetermined coefficients $B_{m n}$ and making them equal to zero, the submatrices in the first row of $M_{\mathrm{i}}$ can be obtained as

$$
\begin{aligned}
& \left\{M_{1-1}^{i}\right\}_{S_{1}, T_{1}}=\int_{X c i}^{X d i} \cos \lambda_{a m 1} x \cos \lambda_{a m 2} x \mathrm{~d} x \int_{Y c i}^{Y d i} \cos \lambda_{b n 1} y \cos \lambda_{b n 2} y \mathrm{~d} y, \\
& \left\{M_{1-2}^{i}\right\}_{S_{1}, T_{2}}=\int_{X c i}^{X d i} \cos \lambda_{a m 1} x \cos \lambda_{a m 2} x \mathrm{~d} x \int_{Y c i}^{Y d i} \xi_{1 b}(y) \cos \lambda_{b n 1} y \mathrm{~d} y .
\end{aligned}
$$

The submatrices $M_{1-3}^{i}$ can be derived by replacing $\xi_{1 b}(y)$ in submatrices $M_{1-2}^{i}$ with $\xi_{2 b}(y)$.

$$
\left\{M_{1-4}^{i}\right\}_{S_{1}, T_{4}}=\int_{X c i}^{X d i} \xi_{1 a}(x) \cos \lambda_{a m 1} x \mathrm{~d} x \int_{Y c i}^{Y d i} \cos \lambda_{b n 1} y \cos \lambda_{b n 2} y \mathrm{~d} y .
$$

The submatrices $M_{1-5}^{i}$ can be derived by replacing $\xi_{1 a}(x)$ in submatrices $M_{1-5}^{i}$ with $\xi_{2 a}(x)$.

$$
\begin{gathered}
\left\{M_{1-6}^{i}\right\}_{S_{1}, T_{6}}=0, \\
\left\{M_{1-7}^{i}\right\}_{S_{1}, T_{7}}=0, \\
\left\{M_{1-8}^{i}\right\}_{S_{1}, T_{8}}=0, \\
\left\{M_{1-9}^{i}\right\}_{S_{1}, T_{9}}=0, \\
\left\{M_{1-10}^{i}\right\}_{S_{1}, T_{10}}=0 .
\end{gathered}
$$

The stiffness and mass matrix of the whole plate $K$ and $M$ can be obtained by replacing the upper limits $(X d i, Y d i)$ and the lower limits $(X c i, Y c i)$ in the above equations with $(a, b)$ and $(0,0)$, respectively.

\section{Data Availability}

The data used to support the findings of this study are available from the corresponding author upon request.

\section{Conflicts of Interest}

The authors declare that they have no conflicts of interest.

\section{Acknowledgments}

The authors gratefully acknowledge the financial support from the National Natural Science Foundation of China (nos. 51705071, 51775125, and 51475088), the Natural Science Foundation of Jilin Province of China (Grants nos. 20190103058JH, 20190201109JC, and 20140101065JC), the
Fundamental Research Funds for the Central Universities of China (no. HEUCFG201713), and the Research Foundation of Education Department of Jilin Province, China (no. 2015244).

\section{References}

[1] D. R. Avalos, H. A. Larrondo, P. A. A. Laura et al., "Transverse vibrations of simply supported rectangular plates with rectangular cutouts carrying an elastically mounted concentrated mass," Journal of Sound and Vibration, vol. 202, no. 4, pp. 585-592, 1997.

[2] D. R. Avalos and P. A. A. Laura, "Transverse vibrations of simply supported rectangular plates with two rectangular cutouts," Journal of Sound and Vibration, vol. 267, no. 4, pp. 967-977, 2003.

[3] K. Y. Lam, K. C. Hung, and S. T. Chow, "Vibration analysis of plates with cutouts by the modified Rayleigh-Ritz method," Applied Acoustics, vol. 28, no. 1, pp. 49-60, 1989.

[4] M. K. Kwak and S. Han, "Free vibration analysis of rectangular plate with a hole by means of independent coordinate coupling method," Journal of Sound and Vibration, vol. 306, pp. 12-30, 2007.

[5] A. Z. Lu, Z. Xu, and N. Zhang, "Stress analytical solution for an infinite plane containing two holes," International Journal of Mechanical Sciences, vol. 128-129, pp. 224-234, 2017.

[6] S. Takahashi, "Vibration of rectangular plates with circular holes," Journal of the Japan Society of Mechanical Engineers, vol. 4, no. 1, pp. 380-385, 1958.

[7] P. Paramasivam, "Free vibration of square plates with square openings," Journal of Sound and Vibration, vol. 30, no. 2, pp. 173-178, 1973.

[8] R. Ali and S. J. Atwal, "Prediction of natural frequencies of vibration of rectangular plates with rectangular cutouts," Computers \& Structures, vol. 12, no. 6, pp. 819-823, 1980.

[9] J. Zhang, T. Li, and X. Zhu, "Free vibration analysis of rectangular Fgm plates with a cutout," Earth and Environmental Science, vol. 283, Article ID 012037, 2019.

[10] N. S. Bardell, R. S. Langley, and J. M. Dunsdon, "On the free in-plane vibration of isotropic rectangular plates," Journal of Sound and Vibration, vol. 191, pp. 459-467, 1996. 
[11] Y. Chen, G. Jin, and Z. Liu, "Flexural and in-plane vibration analysis of elastically restrained thin rectangular plate with cutout using Chebyshev-Lagrangian method," International Journal of Mechanical Sciences, vol. 89, pp. 264-278, 2014.

[12] D. J. Gorman, "Free in-plane vibration analysis of rectangular plates with elastic support normal to the boundaries," Journal of Sound and Vibration, vol. 285, pp. 941-966, 2005.

[13] D. J. Gorman, "Free in-plane vibration analysis of rectangular plates by the method of superposition," Journal of Sound and Vibration, vol. 272, pp. 831-851, 2004.

[14] C. Zhang, G. Jin, Z. Wang et al., "Dynamic stiffness formulation for free vibration of truncated conical shell and its combinations with uniform boundary restraints," Shock and Vibration, vol. 2021, Article ID 6655035, 20 pages, 2021.

[15] D. T. Huang, "Effects of constraint, circular cutout and inplane loading on vibration of rectangular plates," International Journal of Mechanical Sciences, vol. 68, pp. 114-124, 2013.

[16] K. M. Liew, S. Kitipornchai, and A. Y. T. Leung, "Analysis of the free vibration of rectangular plates with central cut-outs using the discrete Ritz method," International Journal of Mechanical Sciences, vol. 45, pp. 941-959, 2003.

[17] I. Shufrin and M. Eisenberger, "Semi-analytical modeling of cutouts in rectangular plates with variable thickness free vibration analysis," Applied Mathematical Modeling, vol. 40, pp. 6983-7000, 2016.

[18] Y. Zhou, Q. Wang, D. Shi et al., "Exact solutions for the free in-plane vibrations of rectangular plates with arbitrary boundary conditions," International Journal of Mechanical Sciences, vol. 130, pp. 1-10, 2017.

[19] E. Ahad Fazl, Q. Wang, Q. Liang, and D. Shi, "A unified solution for free in-plane vibration of orthotropic circular, annular and sector plates with general boundary conditions," Applied Mathematical Modelling, vol. 40, no. 21-22, pp. 9228-9253, 2016.

[20] Z. Shi, X. Yao, F. Pang et al., "A semi-analytical solution for inplane free vibration analysis of functionally graded carbon nanotube reinforced composite circular arches with elastic restraints," Composite Structures, vol. 182, pp. 420-434, 2017.

[21] S. Shi, B. Xiao, G. Jin et al., "Modeling and simulation of transverse free vibration analysis of a rectangular plate with cutouts using energy principles," Shock and Vibration, vol. 2018, Article ID 9609745, 16 pages, 2018.

[22] J. Du, "An analytical method for the in-plane vibration analysis of rectangular plates with elastically restrained edges," Journal of Sound and Vibration, vol. 306, pp. 908-927, 2007. 